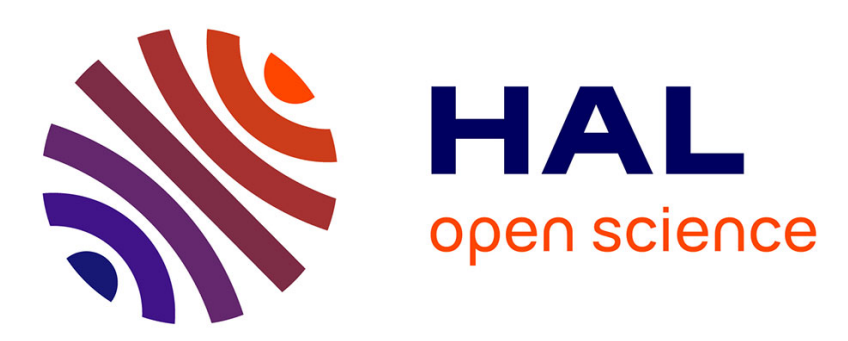

\title{
Numerical investigation of channel blockage by flowing microparticles
}

Gbedo Constant Agbangla, Éric Climent, Patrice Bacchin

\section{To cite this version:}

Gbedo Constant Agbangla, Éric Climent, Patrice Bacchin. Numerical investigation of channel blockage by flowing microparticles. Computers and Fluids, 2014, vol. 94, pp. 69-83. 10.1016/j.compfluid.2014.01.018 . hal-00968541

\section{HAL Id: hal-00968541 \\ https://hal.science/hal-00968541}

Submitted on 1 Apr 2014

HAL is a multi-disciplinary open access archive for the deposit and dissemination of scientific research documents, whether they are published or not. The documents may come from teaching and research institutions in France or abroad, or from public or private research centers.
L'archive ouverte pluridisciplinaire HAL, est destinée au dépôt et à la diffusion de documents scientifiques de niveau recherche, publiés ou non, émanant des établissements d'enseignement et de recherche français ou étrangers, des laboratoires publics ou privés. 


\section{Open Archive Toulouse Archive Ouverte (OATAO)}

OATAO is an open access repository that collects the work of Toulouse researchers and makes it freely available over the web where possible.

This is an author-deposited version published in: http://oatao.univ-toulouse.fr/ Eprints ID: 11194

To link to this article : DOI:10.1016/j.compfluid.2014.01.018

http://dx.doi.org/10.1016/j.compfluid.2014.01.018

\section{To cite this version:}

Agbangla, Gbedo Constant and Climent, Eric and Bacchin, Patrice Numerical investigation of channel blockage by flowing microparticles. Computers \& Fluids . 


\title{
Numerical investigation of channel blockage by flowing microparticles
}

\author{
Gbedo Constant Agbangla ${ }^{\mathrm{a}, \mathrm{b}, \mathrm{e}}$, Éric Climent ${ }^{\mathrm{c}, \mathrm{d}, \mathrm{e}, *}$, Patrice Bacchin ${ }^{\mathrm{a}, \mathrm{b}, \mathrm{e}}$ \\ a Université de Toulouse, INPT, UPS Laboratoire de Génie Chimique, 118 Route de Narbonne, F-31062 Toulouse, France \\ ${ }^{\mathrm{b}}$ CNRS, UMR 5503, F-31062 Toulouse, France \\ 'Université de Toulouse, INPT, UPS, Institut de Mécanique des Fluides, Allée Camille Soula, F-31400 Toulouse, France \\ ${ }^{\mathrm{d}}$ CNRS, UMR 5502, F-31400 Toulouse, France

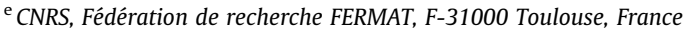

Keywords:

Numerical modeling

Suspension

Particle adhesion

Colloidal forces

Aggregation

Clogging

\begin{abstract}
A B S T R A C T
The dynamic formation of 3D structures of microparticle aggregates blocking the flow through straight microchannels is investigated by direct numerical simulation of the coupled motion of particles and fluid. We use the Force Coupling Method to handle simultaneously multibody hydrodynamic interactions of confined flowing suspension together with particle-particle and particle-wall surface interactions leading to adhesion and aggregation of particles. The basic idea of the Force Coupling Method relies on a multipole expansion of forcing terms (added to the Navier-Stokes equations) accounting for the velocity perturbation induced by the presence of particles in the fluid flow. When a particle reaches the wall or an attached particle, we consider that the adhesion is irreversible and this particle remains fixed. We investigate the kinetics of the microchannel blockage for several solid volumetric concentrations and different surface interaction forces. Many physical quantities such as the temporal evolution of the bulk permeability, capture efficiency, modification of the fluid flow and forces acting on attached particles are analyzed. We show that physical-chemical interactions, modeled by DLVO forces, are essential features which control the blockage dynamics and aggregate structure.
\end{abstract}

\section{Introduction}

The physics of transport, deposition, detachment and reentrainment of colloidal particles suspended in a fluid are of major interest in many areas of fluids engineering: fouling of heat exchangers, contamination of nuclear reactors, plugging of filtration membranes and occlusion of human veins, deposits in microelectronics and in the paper industry. In many solid/liquid separation processes such as micro-filtration or ultrafiltration of water, the limitation of the process performance is related to the fouling of filtration devices. To prevent or control the occurrence of fouling, it is necessary to achieve a better understanding of the respective roles of physical-chemical phenomena and hydrodynamic interactions in a confined suspension of particles. Non-hydrodynamic surface interactions and the adhesion of particles onto solid surfaces are the essential features to be modeled. However, mainly because of a complex interplay between the

\footnotetext{
* Corresponding author at: Université de Toulouse, INPT, UPS, Institut de Mécanique des Fluides, Allée Camille Soula, F-31400 Toulouse, France. Tel.: +33 5 343228 86; fax: +33 534322994 .

E-mail addresses: gbedoconstant@yahoo.fr (G.C. Agbangla), eric.climent@imft.fr (É. Climent), bacchin@chimie.ups-tlse.fr (P. Bacchin).
}

hydrodynamics of the flow, the physical-chemical properties of the filtered suspensions (often in a colloidal state) and the nature of the solid material, predicting fouling dynamics is still challenging.

Different experimental techniques and numerical approaches have been developed to achieve new insights on the local structure of particle aggregates and the kinetics of blockage. Sharp and Adrian [4], by means of experiments in microtubes, observed blockage due to arch formation. The experiments were performed using liquids seeded with polystyrene beads at low volumetric concentration. They showed that a stable balance between the hydrodynamic forces and contact forces (mainly solid friction) between particles and the wall provokes the formation of arches. Wyss et al. [5] studied PDMS microchannels clogging by an aqueous suspension of monodisperse polystyrene beads. The formation of plugs at the microchannel entrance occurred when a critical number of particles had flowed through the pore whatever the flowrate and the particle volume fraction. In this case, the mechanism of blockage was mainly due to successive depositions of particles on walls or interceptions by attached particles. This is drastically different from the bridging mechanism occurring in a stable suspension. Bridging phenomenon is characterized by simultaneous adhesion of several particles. A critical flowrate 


\section{Nomenclature}

FCM and particle dynamics

a particle radius, $m$

$V \quad$ particle velocity, $\mathrm{m} \mathrm{s}^{-1}$

$\rho_{f} \quad$ density of the fluid, $\mathrm{kg} \mathrm{m}^{-3}$

$\mu_{f} \quad$ dynamic viscosity of the fluid, Pa s

$p \quad$ pressure in the fluid, $\mathrm{Pa}$

$\mathbf{u} \quad$ fluid velocity, $\mathrm{m} \mathrm{s}^{-1}$

$\mathbf{x}$ position in the fluid, $\mathrm{m}$

$\Delta$ and $\Delta^{\prime}$ Gaussian envelops of momentum source terms, $\mathrm{m}^{-3}$

$\sigma_{M}$ and $\sigma_{D}$ width of the Gaussian envelops, $m$

$\mathbf{S}_{i j}^{(n)} \quad$ symmetric part of the dipole tensor, $\mathrm{N} \mathrm{m}$

$\mathbf{A}_{l j}^{(n)} \quad$ anti-symmetric part of the dipole tensor $\mathrm{N} \mathrm{m}$

$d^{3} \mathbf{x} \quad$ elementary volume, $\mathrm{m}^{3}$

$F^{(n)} \quad$ monopole force due to the $n$th particle, $N$

$Y^{(n)} \quad$ position of the $n$th particle, $m$

$V^{(n)} \quad$ velocity of the $n$th particle, $\mathrm{m} \mathrm{s}^{-1}$

$\Omega^{(n)} \quad$ rate of rotation of the $n$th particle, $\operatorname{rad~s}^{-1}$

$F_{a} \quad$ attractive force between two particles, N

$F_{r} \quad$ repulsive force between two particles, $\mathrm{N}$

$A_{h} \quad$ Hamaker constant, J $\epsilon_{0} \epsilon_{r} \quad$ fluid permittivity, $C^{2} \mathrm{~J}^{-1} \mathrm{~m}^{-1}$

$\psi \quad$ electrical potential surface, $\mathrm{V}$

$k \quad$ inverse of the Debye length $\mathrm{m}^{-1}$

$z \quad$ distance between the center of two particles, $\mathrm{m}$

$F_{\alpha} \beta \quad$ non-overlapping repulsion force, $\mathrm{N}$

$F_{\text {adh }}$ adhesion force, $\mathrm{N}$

$T_{\text {adh }} \quad$ adhesion torque, $\mathrm{kg} \mathrm{m}^{-2} \mathrm{~s}^{-2}$

W Stokes velocity, $\mathrm{m} \mathrm{s}^{-1}$

$t_{s} \quad$ diffusion time, $\mathrm{s}$

$F_{h} \quad$ hydrodynamic force, $\mathrm{N}$

$F_{\mathrm{pp}} \quad$ repulsive force between particles, $\mathrm{N}$

$F_{\mathrm{pw}} \quad$ particle-wall repulsive force, $\mathrm{N}$

\section{Suspension statistics}

A area of simulation domain, $\mathrm{m}^{2}$

$\phi, \mathrm{C} \quad$ volumetric concentration of particles

$N_{c} \quad$ number of fixed particles onto one wall of area $A$

$N_{p} \quad$ initial number of particles corresponding to $\phi_{0}$

$k$ bulk permeability of channel, $\mathrm{m}^{2}$

$K(\phi) \quad$ dimensionless coefficient forces the particles to overcome the repulsive potential barrier leading to sudden formation of a plug at the pore entrance. This mechanism has been clearly identified by Ramachandran and Fogler [6] when they studied the conditions under which multilayer deposition occurs in a microchannel. In a previous experimental work [10], we demonstrated with microfluidic experiments that very different clogging structures (arches, deposit, dendrites) can be observed in flows of micrometric particles in microchannels. These different structures depend on the hydrodynamic conditions, the particle concentration and the surface interaction magnitude. These features are possibly related to the collective effect of particles (due to multibody particle interactions) and highlights the need for numerical approaches to depict the complex interplay between transport phenomena and multibody interactions.

Although numerical simulations of these phenomena are not widespread, hydrodynamic forces and physical-chemical interactions are more and more frequently taken into account in the modeling and/or simulation of multiphase flows. For examples, Henry et al. [38] used a new Lagrangian stochastic approach to confirm that clogging may result from the competition between particlefluid, particle-surface and particle-particle interactions. Particle deposition can lead to the formation of either a single monolayer or multilayers depending on hydrodynamical conditions, fluid characteristics (such as the ionic strength) as well as particle and substrate properties (such as zeta potentials). For direct numerical simulation of small colloidal particle collision and agglomeration in turbulent flow, Mohaupt et al. [39] proposed a new probabilistic approach. This is based on evaluating continuous relative trajectories between possible collision partners to evaluate the probability for this trajectory to reach the minimum distance corresponding to particle collision. This approach can address new physical issues related to two-phase flow modeling and opens interesting avenues for the simulation of particle interactions, aggregates over a wide range of suspension characteristics.

For aerosols, Marshall [7] investigated the adhesion of particle aggregates onto straight channel walls under laminar flow. To show that particle deposition onto walls is dominated by particle-particle and particle-wall surface interactions, he used a Discrete Element Method assuming that the particle density is much larger than the fluid density. The trajectory equation accounts for Van der Waals and collision forces (soft-sphere model). The translational velocity and rotation rate of each particle are obtained by numerical integration of Newton's second law. They neglected the perturbation of the fluid flow due to the presence of attached particles which limits their study to the early instants of blockage when the permeability of the channel is unaffected by the growing plug. This is a serious shortcoming of this approach. Concerning colloidal suspensions, Tanaka and Araki [8] studied the importance of interparticle hydrodynamic interactions during aggregation using the Fluid Particle Dynamics method. In the FPD method, colloidal suspension is modeled as a mixture of viscous undeformable fluid particles and a non-viscous simple liquid, instead of treating colloids as solid particles. This method is based on a hybrid model, which combines a lattice simulation for continuous fields and an off-lattice simulation for particles. The importance of interparticle hydrodynamic interactions in aggregation, gel formation, and phase separation of colloidal suspensions has been highlighted.

In our study of channel blockage by adhesion of spherical particles, we choose to use the Force Coupling Method to simulate the adhesion and aggregation of spherical particles in laminar channel flow accounting for hydrodynamic interactions and DLVO forces related to the presence of fixed and flowing particles. The paper is organized as follows. The Force Coupling Method and its validation are presented in Section 1. In Section 2, we describe the configuration and conditions of the simulation. The kinetics of clogging according to different volume fraction of particles and interaction forces is investigated in Sections 3 and 4, respectively. The evolution of the bulk permeability, the capture efficiency in the microchannel, the particulate microstructure and the modification of the fluid flow around attached particles will be discussed. Conclusions are drawn in Section 5.

\section{Numerical modeling of flow particle interactions}

Early numerical methods for discrete particle tracking were often based on the restrictive assumption that the presence of particles does not modify the carrying fluid flow (one-way coupling 
Discrete Element Method). This is generally associated with the assumption of point particles [21]. Velocity field and its gradients at the position of the particle center are needed to compute the force balance and finally trajectory equations. Models of collision can be explicitly included to simulate interactions and momentum exchange between particles [7]. Later, these approaches were complemented by adding momentum source terms in Navier-Stokes equations as a model of two-way coupling interactions. These models are able to simulate collective effect of particles on the flow (modification of the bulk density and viscosity) but neglect direct hydrodynamic interactions between particles. The particle size is much smaller than the grid spacing used to compute the fluid flow. This individual particle tracking provides some specific information (velocity, position, trajectory...) for each particle which can be statistically analyzed to provide information on macroscopic modeling.

The theoretical background of the Force Coupling Method is based on particular properties of Stokes equations although this method can also be used at low but non-zero Reynolds numbers. We can note that specific numerical methods have been developed for solving Stokes equations. In the Boundary Element Method (BEM) [12], the surface of each particle is discretized and the linearity of Stokes equations is used to reduce the problem to a set of linear equations involving the unknowns on the sphere surface. The inherent difficulty occurs when two spheres come close to contact. Lubrication forces between these two spheres may not be accurately captured. BEM can easily be extended to non-spherical or deformable particles.

Another method suitable for Stokesian suspension is based on the reflection algorithm [13]. The flow field around each sphere is obtained by summation of cumulative terms related to the presence of nearby spheres. The presence of a particle causes a perturbation flow field which is then reflected onto all other spheres iteratively. These methods are only suitable for small numbers of spherical particles and converge quickly when the spheres are well-separated but for specific arrangements a low rate of convergence may occur.

Multipole methods (Stokesian Dynamics [14,15] has became very popular to simulate suspension dynamics. The flow field is represented in terms of a resistance matrix (extracted from a truncated multipole expansion) relating the external forces and torques on all particles to their velocities and any background flow. The multipole expansion is truncated after the Stresslet (force dipole) and the irreducible quadrupole. Lubrication corrections are added for short separation distance between spheres. The Force Coupling Method stands within this type of approach.

The major advantage of the Force Coupling Method is its flexibility and the modest overhead cost added to an existing $\mathrm{Na}$ vier-Stokes solver (about 10\% for few thousands particles). The FCM can handle spherical or ellipsoidal particles (complex shapes are not possible) moving in a Stokes or finite Reynolds flow including simply the effect of non-hydrodynamic forces together with a good accuracy of multi-body hydrodynamic interactions. This method has been compared to other existing methods for particulate flows (see [40]. The conclusion is that with $6-8$ grid cells within a particle diameter, FCM is very efficient. This allows the simulation of suspension of thousands of particles (for the simulations we performed, around 10,000 particles were flowing through the channel and the total time of simulation was 24 hours on eight $2.8 \mathrm{Ghz}$ processors of a cluster Altix ICE 8200).

\subsection{The Force Coupling Method}

We aim at simulating the trajectories of particle including simultaneously the effect of the carrying flow, direct hydrodynamic interactions between particles and non-hydrodynamic attractionrepulsion forces. The Force Coupling Method (FCM) has been developed for Stokes flow by replacing the Dirac delta function of the standard multipole expansion by a localized force envelope. This avoids the transport of singularities and reproduces the finite size of particles. The FCM has been validated and applied in many configurations of suspension flows; for example, sedimentation problems [16], bimodal suspensions [11]. Multibody hydrodynamic interactions are accounted directly by solving flow equations. The Force Coupling Method can be implemented in any existing flow solver. It is flexible and gives a minor overhead computing time. The translational and angular velocities of a particle are estimated by local averages of the fluid velocity weighted by the corresponding force envelopes. This approach has a computational advantage by reducing the number of grid points necessary to resolve a particle. Typically, it requires only six to eight grid cells to discretize the particle diameter. This is less than other methods, such as the Immersed Boundary Method [20]. Once the resolution is fine enough to resolve the Gaussian force envelope, the FCM can accurately reproduce the flow perturbation. Accuracy and validation tests will be presented hereafter. When two particles are close to contact, the Force Coupling Method underestimates the lubrication effects. As a result, the FCM has been used mainly for volume fractions lower than $20 \%$ and for larger concentration, lubrication corrections must be implemented [17] (see also the study of Yeo and Maxey [35]).

\subsection{Equations of the model}

The FCM has been implemented in JADIM (in-house software developed at IMFT, Institute of Fluid Mechanics, Toulouse) to solve the flow field equations using a finite volume method on a staggered grid. The fluid is incompressible (Eq. (1)) with a constant dynamic viscosity $\mu_{f}$ and fluid density $\rho_{f}$. Incompressibility of the flow is achieved by a fractional step method using an auxiliary potential for the solution of the Poisson equation. The spatial derivatives are computed with second order accuracy and temporal integration is achieved by a third order Runge-Kutta scheme and a semi-implicit Crank-Nicolson scheme for the viscous terms. At low Reynolds numbers, the left hand side of Eq. (2) is negligible and Navier-Stokes equations degenerate to Stokes equations. All particles are represented by forcing terms (Eq. (3)) spatially distributed on a Gaussian envelop (Eq. (4)).

$$
\begin{aligned}
& \nabla \cdot \mathbf{u}=0 \\
& \rho_{f}\left(\frac{\partial \mathbf{u}}{\partial t}+\nabla \cdot \mathbf{u u}\right)=-\nabla p+\mu_{f} \nabla^{2} \mathbf{u}+\mathbf{f}(\mathbf{x}, \mathbf{t})
\end{aligned}
$$

In the FCM, the fluid fills the entire volume of the simulation domain and each particle is represented by forcing terms. In momentum balance Eq. (2), $\mathbf{f}(\mathbf{x}, \mathbf{t})$ accounts for the perturbation induced by the presence of each particle in the suspension. Each $n$ particle (with the total number $N_{a}$ ) acts on the fluid with the force $\mathbf{F}_{1}^{(n)}$ (Eq. (3)).

$$
\mathbf{f}_{i}(\mathbf{x}, \mathbf{t})=\sum_{n=1}^{N_{a}} \mathbf{F}_{i}^{(n)} \Delta\left(\mathbf{X}-\mathbf{Y}^{(n)}(t)\right)
$$

where $\mathbf{Y}^{(n)}$ is the position of the $n$th particle center. The first term of the multipole expansion is called the monopole. It is the finite size analog of the pointwise Stokeslet. This force monopole represents the sum of body force, particle-particle and particle-wall interaction forces (adhesive-repulsive - non-overlapping forces). The finite size of particle (radius a) determines the width of the Gaussian envelop (Eq. (4)) used to spread out source terms on the fluid flow (Eq. (3)). 
$\Delta\left(\mathbf{x}-\mathbf{Y}^{(n)}\right)=\left(2 \pi \sigma_{M}^{2}\right)^{(-3 / 2)} \exp \left(\frac{-\left|\left(\mathbf{x}-\mathbf{Y}^{(n)}\right)\right|^{2}}{2 \sigma_{M}^{2}}\right)$

The relation between the real particle size and the width of the Gaussian envelope is $\sigma_{M}\left(a / \sigma_{M}=\sqrt{\pi}\right)$. This expression is determined analytically to match exactly Stokes drag on an isolated particle translating in a uniform fluid flow $[19,1]$.

The accuracy of the flow streamlines close to the particle surface can be improved by adding to the monopole term, the second term (Eq. (5)) called force dipole. The role of this second term of the multipole expansion is to cancel flow deformation within the volume occupied by the particle (see Lomholt and Maxey [9].

$\mathbf{f}_{i}(\mathbf{x}, \mathbf{t})=\sum_{n=1}^{N_{a}} \mathbf{F}_{i j}^{(n)} \frac{\partial \Delta^{\prime}\left(\mathbf{x}-\mathbf{Y}^{(n)}(t)\right)}{\partial \mathbf{x}_{j}}$

The second forcing term is distributed on a spherical Gaussian envelop (Eq. (6)), as well. The ratio of $a / \sigma_{D}$ is set as to ensure an average zero rate of strain Eq. (7) within the volume occupied by the particle. The width of this Gaussian envelop is given by $\sigma_{D}$ $\left(a / \sigma_{D}=(6 \sqrt{\pi})^{1 / 3}\right)$ which is the analytic expression to verify Eq. (7) for an isolated particle.

$\Delta^{\prime}\left(\mathbf{X}-\mathbf{Y}^{(n)}\right)=\left(2 \pi \sigma_{D}^{2}\right)^{(-3 / 2)} \exp \left(\frac{-\left|\left(\mathbf{X}-\mathbf{Y}^{(n)}\right)\right|^{2}}{2 \sigma_{D}^{2}}\right)$

In the expression of the dipole term, $\mathbf{F}_{l j}^{(n)}$ is a tensor which may be decomposed into symmetric and anti-symmetric parts: $\mathbf{F}_{l \jmath}^{(n)}=\mathbf{S}_{l \jmath}^{(n)}+\mathbf{A}_{l \jmath}^{(n)}$. The symmetric part $\mathbf{S}_{l^{\prime}}^{(n)}$, namely the Stresslet, contributes to enforce a solid body motion (deformation free) within the fluid occupied by the particle. For several particles, an iterative scheme (conjugate gradient with steepest descent scheme) is used to enforce a zero strain rate (Eq. (7)) within the particle volume (see details in the paper by Dance and Maxey [17].

$\frac{1}{2} \int\left(\frac{\partial U_{l}}{\partial X_{j}}+\frac{\partial U_{j}}{\partial X_{l}}\right) \Delta^{\prime}\left(\mathbf{x}-\mathbf{Y}^{n}(n)\right) d^{3} X=0$

The anti-symmetric part $\mathbf{A}_{l j}^{(n)}$ (Eq. (8)) is related to external torque acting on particles yielding rotation of the fluid as a solid body (see Fig. 1).

$\mathbf{A}_{l j}^{(n)}=\frac{1}{2} \epsilon_{l \jmath k} \mathbf{T}_{k j}^{(n)}$

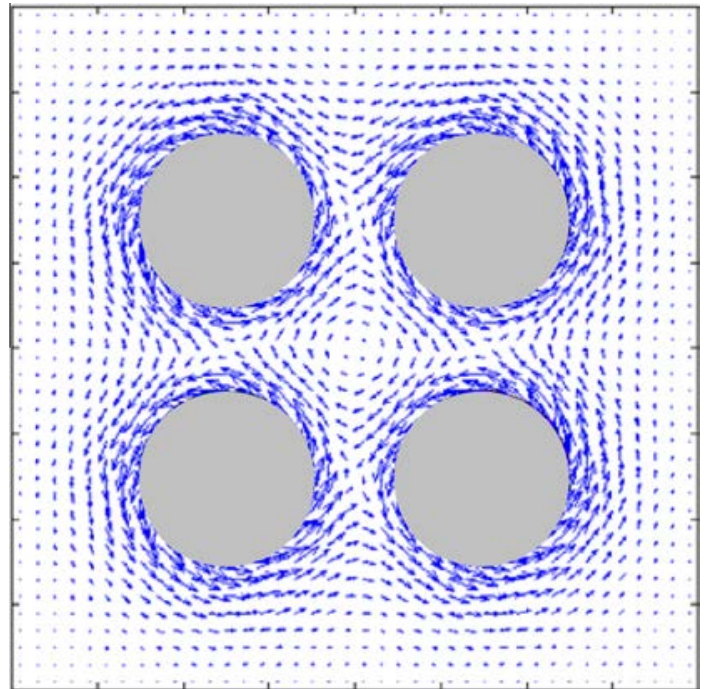

Fig. 1. Schematic view of the rotation of four particles.
Translational and rotational velocities of particles are obtained respectively by spatial averaging of the fluid velocity and vorticity. Velocity (Eq. (9) is integrated over the monopole Gaussian envelop $\Delta$ ) while the fluid vorticity (Eq. (10)) is integrated over the dipole Gaussian envelop $\Delta^{\prime}$.

$\mathbf{V}^{(n)}(t)=\iiint \mathbf{u}(\mathbf{x}, \mathbf{t}) \Delta\left(\mathbf{x}-\mathbf{Y}^{(n)}(t)\right) d^{3} \mathbf{x}$

$\mathbf{\Omega}^{(n)}(t)=\frac{1}{2} \iiint \nabla \times \mathbf{u}(\mathbf{x}, \mathbf{t}) \Delta^{\prime}\left(\mathbf{x}-\mathbf{Y}^{(n)}(t)\right) d^{3} \mathbf{x}$

Finally, the particle trajectory is computed by temporal integration of Eq. (11).

$\frac{d \mathbf{Y}^{(n)}(t)}{d t}=\mathbf{V}^{(n)}(t)$

\subsection{Non-hydrodynamic interaction forces}

The FCM is very flexible and implementing particle-particle or particle-wall forces is straightforward. The surface interactions can be included into the force monopole term. To take into account physical-chemical interactions (adhesion, attractive or repulsive interparticle forces depending on the nature of surface interactions), we choose to use DLVO (Derjaguin, Landau, Verwey and Overbeek) theory as a simple model of non-hydrodynamic forces. This simplified theory can be applied to microparticles flowing in different fluids. Experimentally, repulsion or attraction can be varied by changing solvent properties (electrolyte concentration, $\mathrm{pH}$, ...) or wall and particle material.

\subsubsection{Particle-particle interactions}

In the classic approach, the interaction forces between two rigid bodies are obtained by pairwise summation of the molecular forces. For a pair of spherical and homogeneous particles (radius a), the expression of attractive and repulsive forces are given in Eqs. (12) and (14), see details in Feke and Schowalter [25].

$F_{a}=\frac{4 A_{h}}{3 a}\left(\frac{z}{a}+2\right)\left(\frac{1}{b_{1} b_{2}}-\frac{1}{2}\left(\frac{1}{b_{1}^{2}}+\frac{1}{b_{2}^{2}}\right)\right)$
$b_{1}=\left(\frac{z}{a}\right)^{2}+4 \frac{z}{a}, \quad b_{2}=\left(\frac{z}{a}\right)^{2}+4 \frac{z}{a}+4$
$F_{\mathrm{pp}}=4 \pi \epsilon_{0} \epsilon_{r} \psi^{2} k a\left(e^{-k z}\left(\frac{1-e^{-k z}}{1-e^{-2 k z}}\right)\right)$

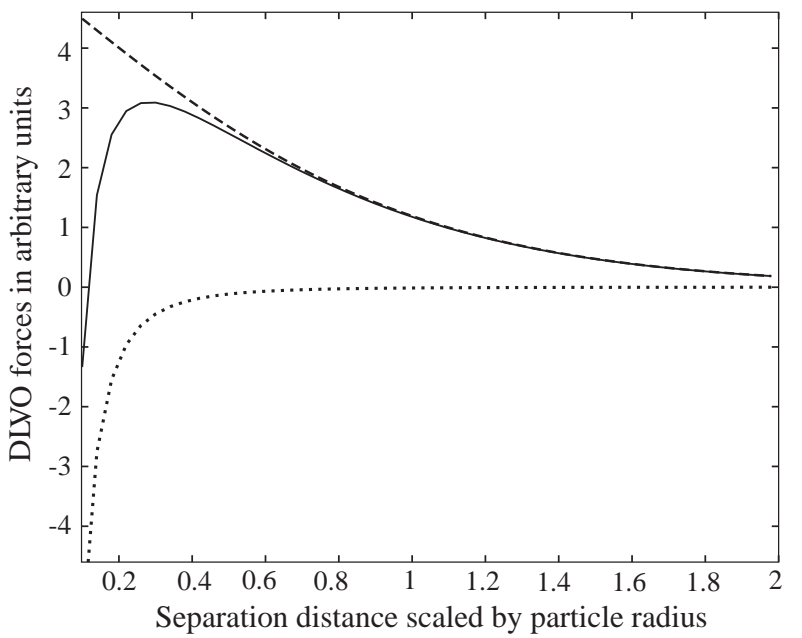

Fig. 2. Profile of DLVO forces: attraction force (dotted line), repulsive force (dashed line), total interparticle force (solid line). 
$A_{h}$ is the Hamaker constant which is related to the properties of the particle material and to the ambient fluid, $z$ represents the distance between the particle surfaces, $k$ is the inverse of Debye length, $\psi$ the zeta potential, $\epsilon_{0}$ the vacuum permittivity and $\epsilon_{r}$ is the relative permittivity of the fluid.

Fig. 2 shows the general shape of DLVO forces for two identical particles. The attractive Van der Waals contribution is a negative force which tends to make particles closer while the electrostatic repulsion force is positive. For the physical-chemical parameters selected in this figure, the sum of the two opposite forces (solid line) shows that repulsion dominates at large separation (suspension is stable and no particle aggregation occurs in a still fluid at low concentration). If the flow (shear flow, streamlines contraction) pushes the particles towards each other and overcome the repulsion barrier, the attractive force will become very strong and yields irreversible adhesion. If three or more particles are involved in DLVO interactions, forces are computed on each particle doublet and pairwise summation is operated.

\subsubsection{Particle-wall interactions}

Regarding particle-wall interactions, Eqs. (15) and (17) are obtained from Eqs. (12) and (14) assuming that one particle radius is infinite. Those expressions are valid provided that the interaction length scale is very small compared to particle radius.

$$
\begin{aligned}
& F_{a}=\frac{A_{h}}{3 a}\left(\frac{1}{b_{1} b_{2}}-\frac{1}{2}\left(\frac{1}{b_{1}^{2}}+\frac{1}{b_{2}^{2}}\right)\right) \\
& b_{1}=\frac{z}{a}, \quad b_{2}=\frac{z}{a}+2 \\
& F_{\mathrm{pw}}=8 \pi \epsilon_{0} \epsilon_{r} \psi^{2} k a\left(e^{-k z}\left(\frac{1-e^{-k z}}{1-e^{-2 k z}}\right)\right)
\end{aligned}
$$

\subsubsection{Non-overlapping force}

During particle interactions, solid surfaces might come in contact due to attractive contribution of DLVO forces. In the FCM, the particles are represented by forcing terms in momentum balance equations. Therefore, particle overlapping must be prevented by a steep repulsion force that could represent forces due to the overlapping of electron clouds (Pauli or Born repulsion). We selected the model (Eq. (18)) proposed by Drazer et al. [26] for short range non-overlapping forces.

$\mathbf{F}_{\alpha \beta}=F_{0} \frac{e^{-z / r_{c}}}{1-e^{z / r_{c}}} \mathbf{e}_{\alpha \beta}$

where $F_{0}$ is a force scale, $z$ is the normalized gap between the surfaces of two particles or between the particle and a wall, $r_{c}$ is the interaction distance and $\mathbf{e}_{\alpha \beta}$ is a unit vector along the line of particle centers $\beta$ to $\alpha$. The value of $F_{0}$ is proportional to $12 \pi \mu_{f} a^{2} \gamma_{0}$, with $\gamma_{0}$ being the wall shear of Poiseuille flow. This is an estimate of the force experienced by a particle coming into contact with a fixed particle attached onto the wall.

\subsection{FCM and resistance formulation}

The formulation of the Force Coupling Method permits to solve mobility problems: forces and torques are imposed to obtain particle velocities, rotation rates and trajectories. However, when a particle touches a wall the adhesion force holds this particle fixed. In that case, the condition which has to be satisfied is zero velocity for the attached particle. This corresponds to a resistance problem: the force acting on a particular particle depends on multibody hydrodynamic interactions and DLVO forces and has to be adequately found to verify the zero velocity condition. This is an important feature of our simulations, particles fixed at the wall are allowed neither to slide relative to the wall nor to be resuspended by the flow (we assume that adhesion is irreversible and a particle touching the wall or an already attached particle remains fixed). To hold a particle fixed, an iterative scheme is used to evaluate each adhesion force and torque. This iterative scheme (Eqs. (19) and (20)) corresponds to solving the resistance problem enforcing the condition of zero velocity and zero rotation rate for attached particles. At each iteration, Navier-Stokes equations are solved to account for multibody hydrodynamic interactions and DLVO forces.

$$
\begin{aligned}
& \frac{d^{*} \mathbf{F}_{\mathrm{adh}}\left(t^{*}\right)}{d t^{*}}=-\alpha_{1} \mathbf{V}\left(t^{*}\right) \\
& \frac{d^{*} \mathbf{T}_{\mathrm{adh}}\left(t^{*}\right)}{d t^{*}}=-\alpha_{2} \mathbf{\Omega}\left(t^{*}\right)
\end{aligned}
$$

$\alpha_{1}$ and $\alpha_{2}$ are numerical penalty parameters which are properly selected to reduce the number of iterations. When the velocity $\mathbf{V}\left(t^{*}\right)$ and the rotation $\boldsymbol{\Omega}\left(t^{*}\right)$ reach the threshold of convergence (typically $10^{-5}$ ) for all fixed particles, the forces (Eq. (19)) and torques (Eq. (20)) of adhesion are then known and the simulation proceeds to a new time step of particles and fluid motion.

Fig. 3 is a schematic presentation of the algorithm of the Force Coupling Method (FCM) over one time step.

\subsection{FCM validation tests}

The validity of the Force Coupling Method has already been verified extensively (see for example Lomholt et al. [18] and Abbas et al. [11] and references therein).

\subsubsection{Drag and torque on a single particle held fixed in a Poiseuille flow}

We investigate the basic configuration of a single particle in a Poiseuille flow and its hydrodynamic interaction with the wall. This is of particular interest for our study of flowing suspension in microchannel (laminar regime at low Reynolds number). Using the iterative scheme with FCM (resistance problem), we determine the evolution of the force $\left(F^{P}=6 \pi \mu U a \lambda^{P}\right)$ and torque $\left(T^{P}=8 \pi \mu U a^{2} \lambda_{T}^{P}\right)$ acting on the particle held fixed at different separation distances from the wall. This test is similar to Lomholt et al. [18] and it validates our implementation of the FCM for resistance problems in our JADIM code (figures are not reported in the present paper). The dimensionless $\operatorname{drag} \lambda^{P}$ and torque $\lambda_{T}^{P}$ coefficients are compared to the results obtained by Ganatos et al. [22]. Our simulation results are in good agreement with Lomholt et al. [18]. The effect of the dipole Stresslet is important for separation distance shorter than a quarter radius. For such distances, DLVO forces become dominant and may screen hydrodynamic effect. Only the monopole term will be considered in the following tests and in the simulation of channel clogging. Using the monopole term only, the drag force acting on the fixed particle provokes a long range perturbation within the channel flow (yielding bulk permeability reduction) which will interact with the other particles of the suspension.

\subsubsection{Settling velocity of a particle in a periodic array}

We consider the configuration of a single settling particle with tri-periodic boundary conditions as a simple model of porous media (the size of the domain varies the particle volume fraction $C$ or equivalently porosity). We investigate in the low Reynolds regime the flow through a regular array of particles of length $L$ (Fig. 4). The particle experiences a constant body force (buoyancy force $F$ in the monopole term) and the FCM gives the settling speed $V$. Several studies have already investigated this simple configuration either theoretically or numerically. Sangani and Acrivos [23] determined for a periodic cubic lattice the evolution (Eq. (21)) of 


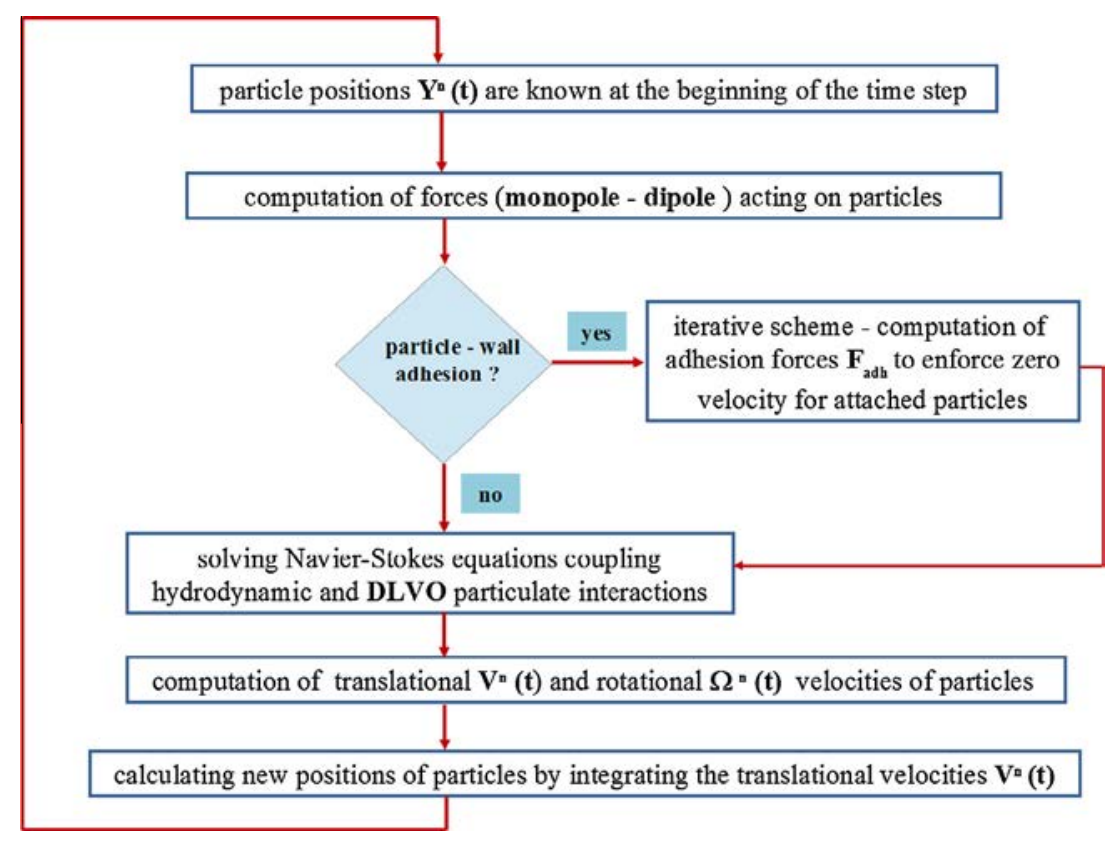

Fig. 3. Detailed algorithm of the FCM.

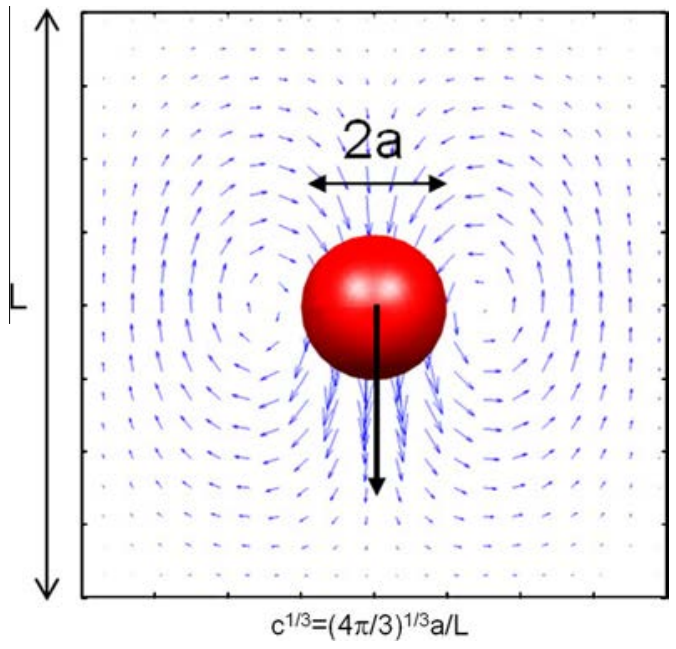

Fig. 4. A settling sphere in a tri-periodic cubic array of particles.

the sedimentation velocity. The settling speed is scaled by Stokes velocity $W$ in a quiescent infinite fluid.

$\frac{V}{W}=1-1.7601 C^{1 / 3}+C-1.5593 C^{2}+3.9799 C^{8 / 3}-3.0734 C^{10 / 3}+O\left(C^{11 / 3}\right)$

$C=\frac{4 \pi a^{3}}{3 L^{3}}$

In Eq. (22) $C$ is the volumetric fraction of particles, $L$ is equal to the length of the periodic domain and $W=F / 6 \pi \mu a$ is the Stokes settling velocity for a single sphere when $L \gg a$.

The characteristic distance $L / a$ between particles in the cubic array is proportional to $C^{-1 / 3}$. Simulation results are presented in Fig. 5 and compared to the analytical prediction of Sangani and Acrivos [23]. The normalized sedimentation velocity decreases when the volume fraction increases. A good agreement is obtained for $C^{1 / 3} \leqslant 0.6$ (or $C \leqslant 20 \%$ ). For $C^{1 / 3} \geqslant 0.6$, the short range hydrodynamic interaction comes into play and the effect of the

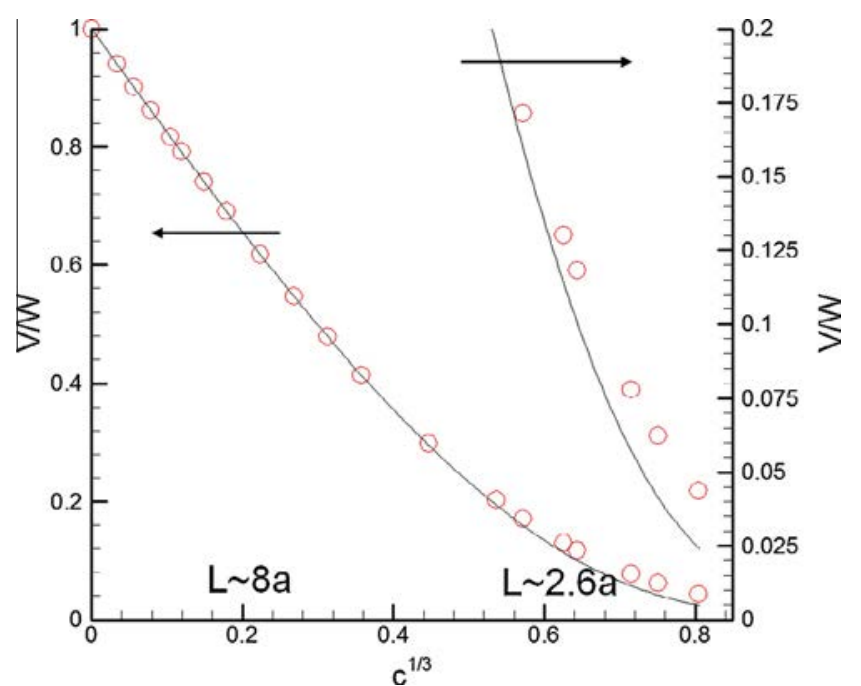

Fig. 5. Evolution of the settling velocity $V$ of a particle in a periodic cubic array vs volume fraction $C$. $V$ is scaled by the Stokes settling velocity $W$ of a sphere in infinite fluid. - results of Sangani and Acrivos [23], o results obtained with the FCM [19] monopole term only. $C^{1 / 3}<0.6$ left axis and $c^{1 / 3} \geqslant 0.6$ close up of the data (see right axis).

monopole term needs to be complemented with dipole and lubrication corrections.

\subsubsection{Interaction between two particles in the vicinity of the wall channel}

We consider the interaction between two particles including DLVO repulsive force and hydrodynamics (one particle is fixed on the wall and the other one is free to move until contact). The channel dimensions $X * Y * Z$ are equal to $28.8 a * 9.6 a * 5.12 a$ in the streamwise, cross stream and spanwise directions, respectively. The effect of non-hydrodynamic forces is varied $\left(\epsilon_{0} \epsilon_{r} \psi^{2}\right)$ while $k a=4$ ( $k$ being the inverse Debye length) is kept constant. The fluid flow is driven by a constant pressure drop along the $X$ direction. This pressure drop induces Poiseuille flow with maximum 
centerline velocity at $Y / 2$. We choose as a hydrodynamic reference force scale $F_{h}$ (Eq. (23)) as follows:

$F_{h}=6 \pi \mu a^{2} \gamma_{0}$

Initially, one particle is fixed onto the wall $\left(X_{p} / a=16\right.$ and $\left.Y_{p} / a=1\right)$. The second particle is free to move through the channel until contact occurs. The starting position $X_{p} / a=6$ and $Y_{p} / a=2$ is kept constant. The only parameter varied in the simulations $\left(\epsilon_{0} \epsilon_{r} \psi^{2}\right)$ is the strength of the particle-particle repulsion force $F_{\mathrm{pp}}$.

Without repulsion force, the moving particle follows the flow streamline (dashed line in Fig. 7) and comes into contact with the fixed particle. Typically, we assume that contact occurs when the separation distance is equal to $a 10^{-5}$. Once at contact, we assume that the particle experiences irreversible adhesion. The Force Coupling Method is formulated to solve mobility problems (force and torque are known to compute the translational and rotational velocities and proceed to trajectory). For the specific case of particle deposition onto a fixed wall, a resistance problem has to be solved: we know that particle velocities are zero but need to find the acting forces. This is achieved by an iterative scheme. The perturbation of the fluid flow obtained after contact of the two attached particles is shown in Fig. 6. In the presence of repulsive forces, the particle trajectory deviates across flow streamlines. This deviation becomes more and more pronounced when the interparticulate repulsive force increases. For $F_{\mathrm{pp}}=F_{h} / 18$, the repulsion force acting on the moving particle is not strong enough to prevent contact (solid line in Fig. 7). In that case, the hydrodynamic forcing overcame the DLVO repulsion barrier. On the contrary, for $F_{\mathrm{pp}}=F_{h} / 9$, the repulsion force is larger than hydrodynamic forcing due to shear. The contact and adhesion are prevented. The particle will continue to flow after a significant deviation of the trajectory (dotted line in Fig. 7).

\subsubsection{Hydrodynamic interactions between an array of fixed particles} and a flowing particle

The configuration proposed by Bhattacharya et al. [24] consists in an array of particles fixed onto a plane wall in a confined channel with dimensions $X=34 a ; Y=2.5 a ; Z=21 a$ respectively in the streamwise, cross-stream and spanwise directions. A single flowing particle is injected. This channel is formed by two infinite plane walls separated by a thin fluid gap $(H=2.5 a)$ in order to mimic highly confined systems. The fluid velocity profile at the inlet is a Poiseuille flow. The array of fixed particles owns two possible exits (see Figs. 8 and 9). One or both of those exits can be blocked by adding an extra attached particles to the wall. The test in this simulation consists in following a single particle through this maze. No repulsion forces are used. Figs. 8 and 9 show different simulation configurations corresponding to different initial positions of the flowing particle. Due to the long range hydrodynamic interaction,

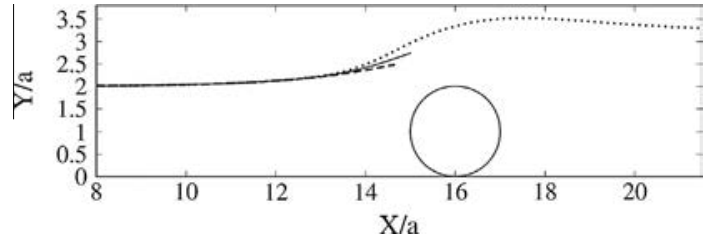

Fig. 7. Different particle trajectories in a 3D channel under Poiseuille flow (normalized velocity $V=4.5$ at $Y / 2$ ). Trajectories are obtained for different values of DLVO repulsive force $F_{\mathrm{pp}} . F_{\mathrm{pp}}=0$ : no repulsion between particles, adhesion occurs (dashed line), $F_{\mathrm{pp}}=F_{h} / 18$ : weak repulsion unable to prevent adhesion of the moving particle onto the fixed one (solid line), $F_{\mathrm{pp}}=F_{h} / 9$ : strong repulsion preventing particle adhesion (dotted line)

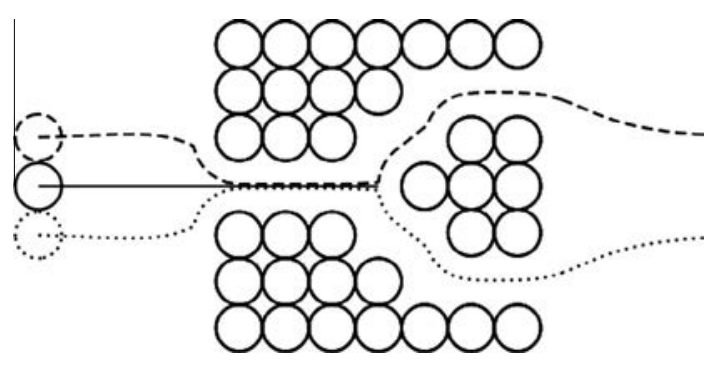

Fig. 8. Particle trajectories through an array of fixed particles. The trajectories are shown in $X-Z$ plane in a confined channel $(H=2.5 a)$ for three different initial positions of the moving particle. The two exits are open. Particle starting in front of the array follows the solid line and cannot leave the particle array. It moves through the center of the array and comes into contact with the central cluster due to the perfect symmetry of the flow. Dashed and dotted lines correspond to symmetric offcentered initial positions.

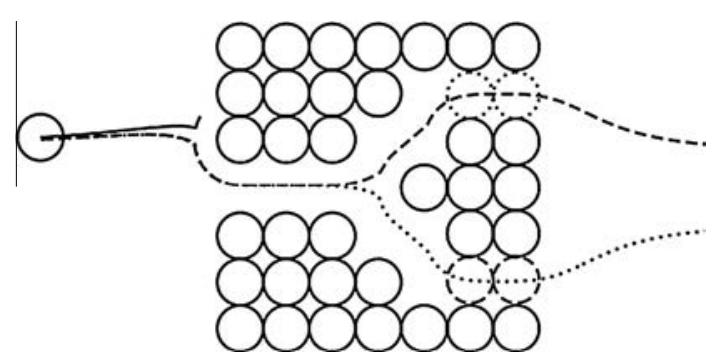

Fig. 9. Particle trajectories through an array of fixed particles. The trajectories are shown in $X-Z$ plane in the confined channel $(H=2.5 a)$ for the same initial position of the moving particle. The two exits can be blocked partially or totally by adding extra fixed particles. Dotted line for the particle trajectory when the upper exit is blocked (dotted circles). Dashed line trajectory when the bottom exit is blocked (dashed circles). Solid line trajectory corresponds to both exits blocked.

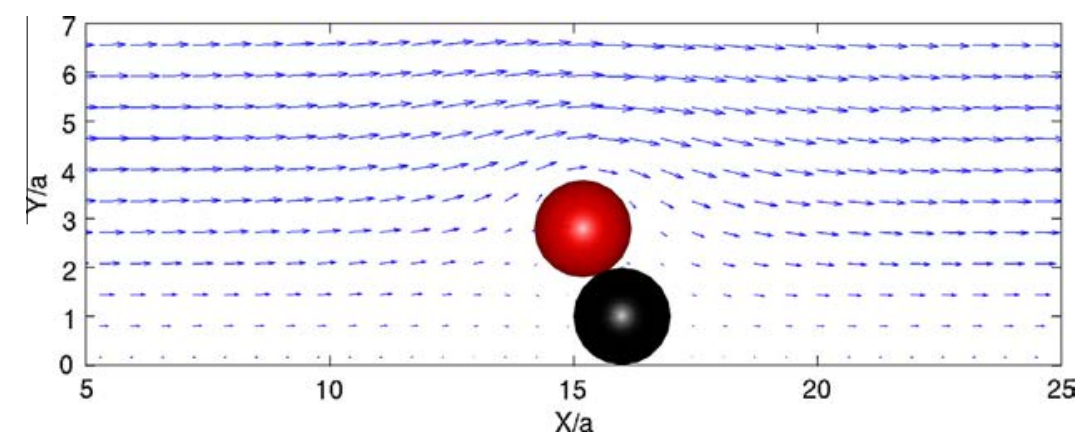

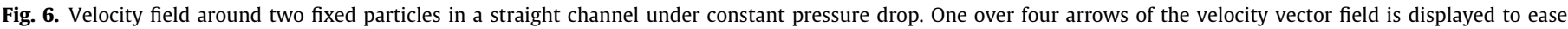
visualization. 
particle trajectories are drastically different if the exits are blocked (the moving particle feels the presence of blocking particles at the entrance of the maze).

Trajectories for different initial positions are presented in Fig. 8 when both exits remain open. Under this simulation configuration, the moving particle enters the particulate maze for any initial position in front of the hole. The choice of the exit depends on its initial position. When the freely moving particle starts perfectly along the axis of symmetry $(Z / 2)$, it cannot leave the particle array. This particle moves through the central part of the array and comes into contact with the cluster due to the perfect symmetry of the flow. When the initial $Z$ value of the free particle is different from $Z / 2$ (off-centered initial position), it moves and leaves the array from the upper exit (dashed line in Fig. 8) or from the bottom exit (dotted line in Fig. 8). This behavior is totally dependent on the initial position.

Other configurations of free particle trajectories are showed in Fig. 9. The exits of the array are blocked partially or totally by adding two or four fixed particles. Trajectories of the particle are compared with the same initial position. When the two exits are blocked, the freely flowing particle does not enter the particle maze (solid line in Fig. 9). It is pushed on one side by the flow (fluid flow is blocked by the particle array in this simulation configuration). This is in agreement with [24]. When a single outlet is blocked, the upstream hydrodynamic interaction forces the free particle to find its way towards the exit. The free particle moves through the array and reaches the exit due to asymmetry in the flow induced by fixed particles.

From these simulations, it is clear that the perturbation of the fluid flow induced by the presence fixed particles onto walls is correctly prescribed by the FCM. All these results are precisely in line with those of Bhattacharya et al. [24] and show that fluid flow perturbations play an important role in the collective dynamics of particles.

\section{Configuration of 3D channel simulation}

The simulation domain (Fig. 10) consists in a 3D channel $(190 \times 40 \times 32$ regularly spaced grid points $)$ of dimensions $61 a \times 15 a \times 10 a$ in the streamwise, cross-stream and spanwise directions. All boundary conditions are noted on the six faces of the domain. No-slip walls are imposed on faces 1 and 3. Faces 2 and 4 are the inlet and outlet sections for the particle trajectories. Finally, periodic boundary conditions are imposed for the fluid flow and particles onto faces 5 and 6 (spanwise $z$ direction). A constant pressure drop is imposed along the channel streamwise direction while periodic boundary conditions are imposed for the flow.

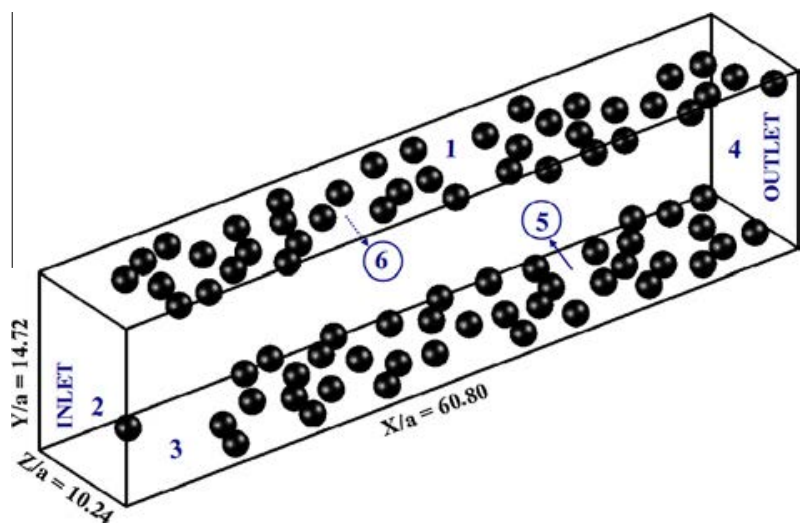

Fig. 10. Schematic view of a plane channel and the corresponding boundary conditions. Some particle are initially attached onto the wall.
Without particles, the pressure drop induces a Poiseuille flow with an average velocity $v_{\text {mean }}$.

All simulations are made dimensionless using the particle radius $a$ as characteristic length scale, the mean flowing velocity $v_{\text {mean }}$ as the reference velocity. Typically, the particle diameter equals $5 \mu \mathrm{m}$ and the channel width $20 \mu \mathrm{m}$ which matches our earlier experimental conditions [10]. Fluid flow is laminar in all simulations (channel Reynolds number is equal $O\left(10^{-2}\right)$ ). Those particles are assumed to be in the high range of colloidal particles (no Brownian motion which is realistic for particles larger than $1 \mu \mathrm{m})$. The particles are neutrally buoyant. Inertia and gravity effects are negligible. Particle diameter corresponds to 8 grid nodes.

Particles are initially seeded at random non-overlapping positions throughout the channel. Several draws (typically 3 ) of the random seeding have been proceeded and the results presented are averages over the three simulations under the same conditions. Particles reaching the outlet are withdrawn from the simulation. New particles (randomly seeded along $Y-Z$ plane) pass through inlet face 2 assuming a constant and uniform concentration of particles and a Poiseuille flow upstream of the simulation domain. To inject new particles in the channel through face 2 , we consider a suspension reservoir where particles move freely along the $x$ direction (no interactions, particles follow straight streamlines with velocity prescribed by the Poiseuille flow). When the streamwise coordinate of a particle corresponds to the entrance of the simulation domain, this particle is injected into the channel and hydrodynamic and DLVO forces are accounted for in the simulation.

Compared to channel and particle widths, the attractive Van der Waals force towards the walls (faces 1 and 3 ) has a very short interaction distance. Therefore, the cross-stream displacement of particles is mainly related to shear induced self-diffusion and the time required for the first particle to attach onto the wall may be very long. Assuming a simple shear flow of strength equal to the Poiseuille wall shear, the diffusion time $t_{s}$ can be estimated with Eq. (24).

$t_{s}=\frac{Y^{2}}{4 D\left(\phi_{0}\right)}$

$Y$ represents the height of the simulation domain and $D(\phi)$ is the self-diffusion coefficient. Self-diffusion (different from Brownian diffusion) occurs in a shear flow due to pairwise (or multibody) hydrodynamic and repulsive interactions. Encounters of particles is induced by the presence of shear which leads finally to crossstream displacement. Based on the work of Abbas et al. [11] which summarizes several studies under Stokes flow, the evolution of $D(\phi)$ can be determined either from numerical simulations or experiments. Therefore, the characteristic diffusion time with a $10 \%$ particle concentration corresponds to dimensionless times ranging from 4000 to 6000 . Our simulations will show that complete clogging of the channel occurs on time scales ten to fifteen times shorter. Therefore, early times of deposition are not simulated because at low to moderate concentration the probability for a particle to touch the wall is low. This corresponds to a very long induction time. To reduce the computation time we fixed a number $N_{c}$ (Eq. (25)) of particles at random locations on each wall assuming uniform spatial distribution of particles.

$\frac{N_{c} \frac{4}{3} \pi a^{3}}{2 a A}=\phi_{0}=\frac{N_{p} \frac{4}{3} \pi a^{3}}{Y A} \Rightarrow N_{c}=\operatorname{Round}\left(N_{p} \frac{2 a}{Y}\right)$

Round is the function which reduces the value of $N_{c}$ down to the nearest integer. $N_{c}$ is the number of fixed particles onto one wall of area $A$ and $N_{p}$ is the initial number of all the particles corresponding to solid volumetric fraction $\phi_{0}$.

Simulations are performed under imposed pressure drop along the channel streamwise direction. We assume that a strong 
adhesion force fixes the particles to the wall or to other attached particles when they come to contact. This contact occurs when the separation distance between two particles is equal to $10^{-5} a$. Once at contact, we assume that the particles becomes adhesive and this adhesion is irreversible. No resuspension or rearrangement of fixed particles within an aggregate is allowed. As the plug is forming, the flowrate decreases progressively. The simulation is stopped when the fluid flowrate has been reduced in a significant way compared to its initial value.

Firstly, the effect of the volumetric concentration of particles (5-10-15-20\%) is analyzed with purely adhesive particles (no DLVO repulsion). Later on, for a particle volume fraction equal to $10 \%$, the effect of non-hydrodynamic interactions is analyzed by considering several magnitudes of the repulsive force barrier between particles.

\section{Channel blockage under pure adhesion}

The major interest of direct numerical simulations is the detailed data generated by the model. All the particulate quantities (position, velocity) and fluid flow field are known at each time step. Those raw data have to be post-processed to analyze relevant quantities which may be compared to experiments, theoretical predictions or for understanding the physics. We have selected a number of macroscopic quantities such as the channel bulk permeability derived from Darcy law, the capture efficiency of the plug and also microstructure description through the coordination number (aggregate particle structure), the distribution of the forces acting on particles and the modification of the fluid flow around attached particles. In this first set of simulations, we fix to zero the repulsive potential of DLVO forces and assume irreversible adhesion.

\subsection{Evolution of channel permeability}

In simulations, the evolution of the channel permeability $k(t)$ accounting for the presence of attached particles will be made dimensionless using the reference permeability $k_{0}$ of the channel at $t=0$ (with only $N_{c}$ particles attached onto the wall).

In Fig. 11, the normalized permeability is plotted as a function of the normalized volume of injected fluid (ratio of injected volume to the simulation domain). We can observe the effect of the inlet particle concentration $\left(\phi_{0}\right)$. A continuous decrease of the normalized permeability is observed while the normalized volume of injected fluid increases: the additional number of attached

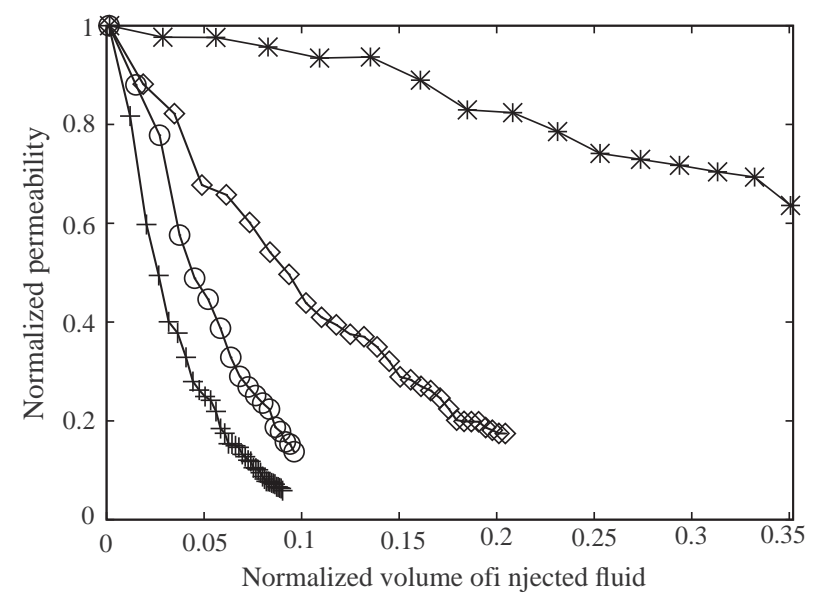

Fig. 11. Evolution of the scaled permeability $v s$. normalized volume of injected fluid for different particle volume fractions: $-*-5 \%,-\diamond-10 \%,-\circ-15 \%,-+-20 \%$. particles increases leading to the progressive clogging of the channel. For an injected volume fraction equal to 0.05 , the normalized permeability is reduced from 0.95 for 45 attached particles $\left(\phi_{0}=5 \%\right)$ to 0.25 for 195 attached particles $\left(\phi_{0}=20 \%\right)$. The permeability reduction is more important for larger volume fraction of particles [31]. It increases more rapidly when particle concentration of the flowing suspension is larger [32]. Fig. 12 shows the rapid reduction of permeability when the particle concentration is larger. At high volume fraction of particles, many new particles are introduced through the channel entrance within a short time, and this leads to the rapid blockage of the flow. Fig. 13 presents the evolution of the normalized permeability as a function of the additional number of attached particles. The volume fraction of the particle suspension has no effect on the permeability evolution when time (or equivalently injected volume) has been replaced by the instantaneous concentration of all attached particles. All the curves corresponding to different inlet concentration collapse on a single evolution. Actually, the same additional number of fixed particles (80) induces a similar reduction of channel permeability (equal to 0.2 ) for any suspension concentration at the inlet. Therefore, we can expect that the same structure of particle aggregates are formed although the channel clogging occurs faster at larger volume fraction of injected suspension.

In Fig. 13, the simulation results are compared to the model of Hermia [2] for a standard clogging mechanism (exponent $n=3 / 2$ in Hermia's model). In such model, the deposited particles on the walls induce a progressive reduction of the channel section: the reduction of the effective channel height is related to the volume occupied by the adhered particles. It is then assumed that the repartition of solid is uniform and leads to a dense thick layer. It is interesting to observe that, for the same additional number of fixed particles in the channel, the normalized permeability decreases faster in the dynamic simulations. It is equal to 0.15 for 320 particles whereas the prediction of Hermia's model is 0.82 . This model would be adequate for much smaller particles which would be attached homogeneously on both walls. In our simulations, fixed particles lead to the formation of 3D loose aggregates at different locations within the domain and cause significant and rapid perturbations of the flow. The plug formed by fixed particles progresses towards the center of the channel where the velocity is high and induces a strong reduction of permeability.

We consider the case corresponding to particles randomly seeded and fixed throughout the channel (static simulations). We compute the reduction of permeability with FCM for this random

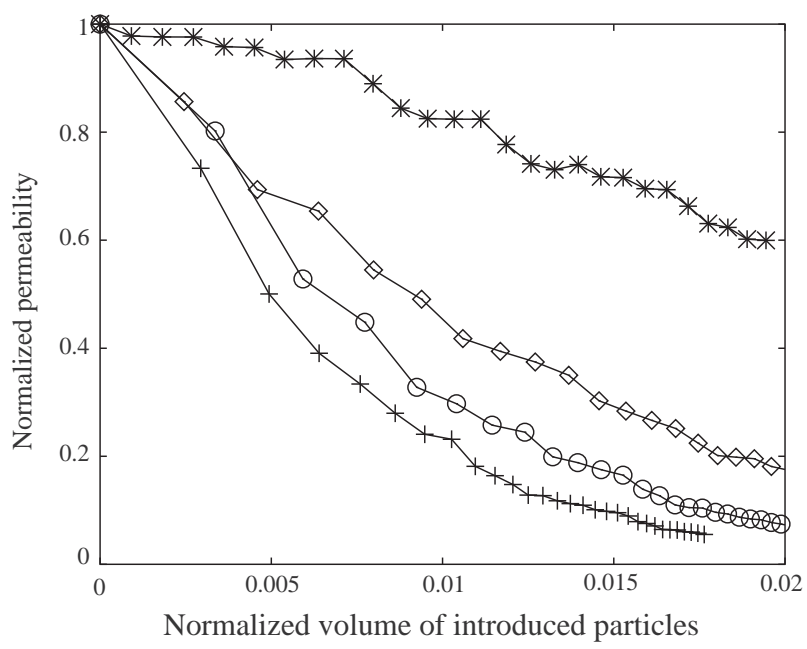

Fig. 12. Evolution of the scaled permeability vs. normalized volume of introduced particles for different particle volume fractions: $-*-5 \%,-\diamond-10 \%,-\circ-15 \%,-+-20 \%$. 


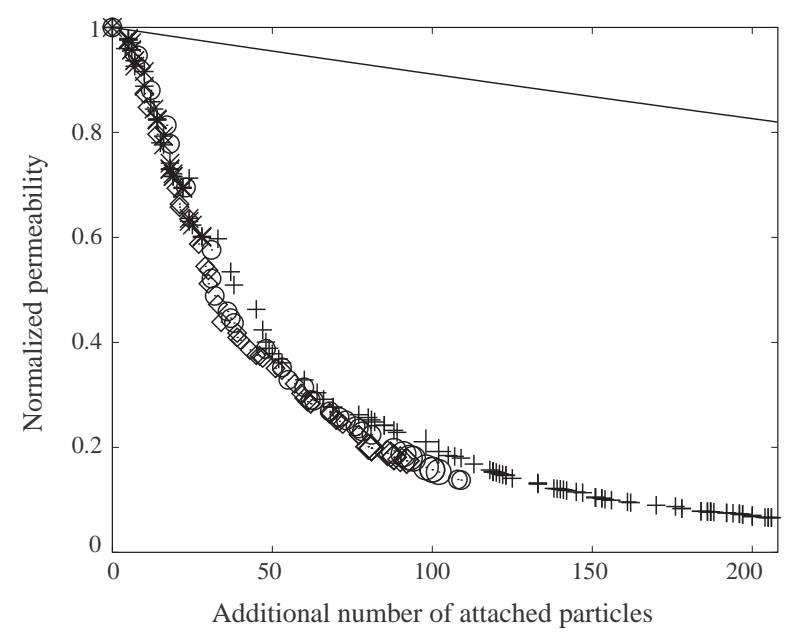

Fig. 13. Evolution of the scaled permeability $v$ s. additional number of attached particles for different particle volume fractions: $* 5 \%, \diamond 10 \%, \circ 15 \%,+20 \%$, Hermia.

arrangement of particles, typically averaged over eight independent seedings. Comparing the permeability evolution for the static simulations to the dynamic cases of the channel clogging (Fig. 14), we note that for similar particle volume fraction $\phi$ (Eq. (26)), the permeability is higher for the dynamic simulations.

$\phi=\frac{\frac{4}{3} \pi a^{3} N_{\text {fixed }}}{V}$

$N_{\text {fixed }}$ is the number of attached particles and $V$ represents the channel volume.

When particles are randomly seeded, they have an equal probability to be located anywhere in the channel while in the dynamic simulation fixed aggregates always start to form close to channel walls. These early attached particles are lying in zones of low fluid velocities and induce weaker reduction of permeability. This point will be confirmed in next section with the study of the aggregate microstructure.

The variation of permeability can also be compared to theoretical (regular arrangement of particles) or empirical permeability models. For these models, we used the approach proposed by Zick and Homsy [27] which relates hindered settling velocity for spheres to the permeability of fixed particles experiencing uniform

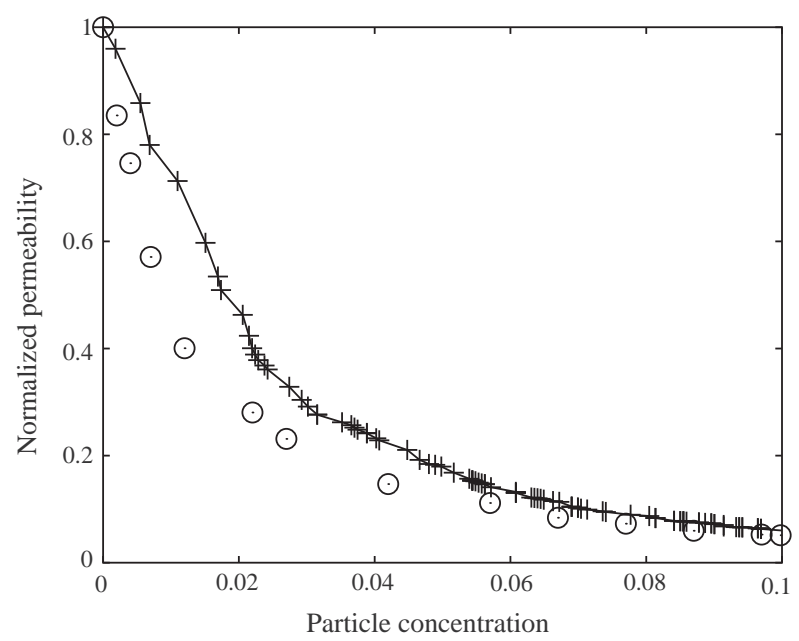

Fig. 14. Evolution of the permeability vs. volume fraction of fixed particles. o Randomly seeded fixed particles, -+- Dynamic simulations leading to blockage. flow. The original expression has been modified to account for the effect of channel walls. We consider that the two walls induce the hydrodynamic resistance equal to $1 / k_{w}$ in absence of particles while the hydraulic resistance induced by the presence of the fixed particles in the simulation domain is equal to $1 / k_{c}$. The total resistance $(1 / k)$ induced by both the presence of particles and walls is given by Eq. (27):

$\frac{1}{k}=\frac{1}{k_{w}}+\frac{1}{k_{c}}$

where $k_{c}=2 a^{2} K(\phi) / 9 \phi$ is the permeability given by Zick and Homsy [27] for uniform flow. The expression of the permeability $k$ is given by Eq. (28):

$$
\begin{aligned}
\frac{1}{k} & =\frac{1}{k_{w}}+\frac{1}{k_{c}} \Rightarrow \frac{1}{k}=\frac{1}{k_{w}}+\frac{9 \phi}{2 a^{2} K(\phi)} \\
\frac{1}{k} & =\frac{2 a^{2} K(\phi)+9 \phi k_{w}}{2 a^{2} k_{w} K(\phi)} \Rightarrow k=\frac{2 a^{2} k_{w} K(\phi)}{2 a^{2} K(\phi)+9 \phi k_{0}} \\
k & =\frac{1}{\frac{1}{k_{w}}+\frac{9 \phi}{2 a^{2} K(\phi)}}
\end{aligned}
$$

Scaling the permeability with $k_{w}$ gives the final expression in Eq. (29):

$$
\frac{k}{k_{w}}=\frac{1}{1+\frac{9 \phi k_{w}}{2 a^{2} K(\phi)}}
$$

$K(\phi)$ represents the dimensionless hindered settling velocity proposed by several authors. We compare our simulation results using Eq. (28) with different laws of $K(\phi)$ : Richardson and Zaki [28], Batchelor (for dilute suspensions $\phi \ll 1$ ) [29], Happel (regular array) [3] and Kim and Russel (random array) [30].

$$
\begin{aligned}
& \text { Richardson-Zaki } K(\phi)=(1-\phi)^{4.5} \\
& \text { Batchelor } K(\phi)=(1-6.55 \phi) \\
& \text { Happel } K(\phi)=\frac{6-9 \phi^{\frac{1}{3}}+9 \phi^{\frac{5}{3}}-6 \phi^{2}}{6+4 \phi^{\frac{5}{3}}}
\end{aligned}
$$

Kim and Russel $K\left(\phi=1+(3 / \sqrt{(2)}) \phi^{\frac{1}{2}}\right.$

$$
+(135 / 64) \phi \log (\phi)+16.456 \phi
$$

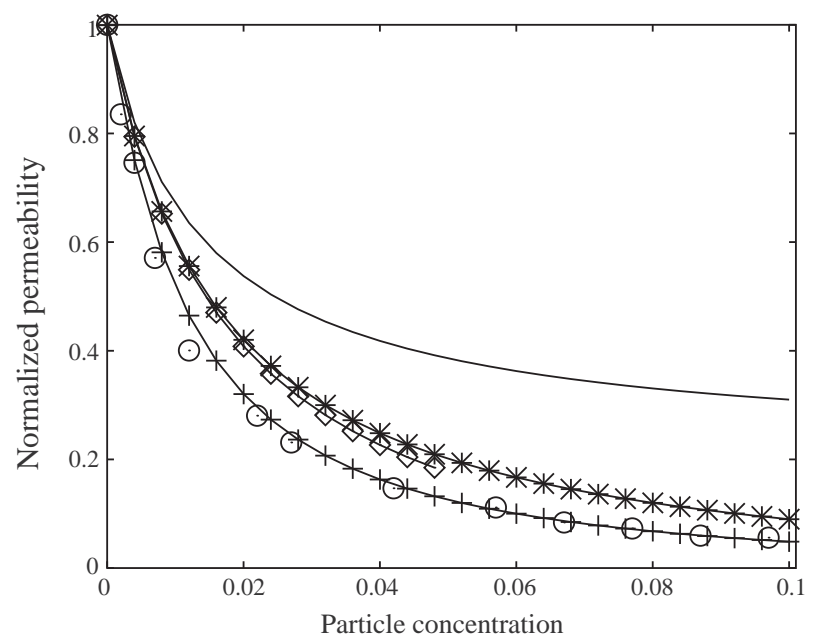

Fig. 15. Evolution of the permeability vs. volume fraction of fixed particles, Kim and Russel, $\quad-*-\quad$ Richardson and Zaki, $\quad-\diamond-\quad$ Batchelor, -+- Happel, 。 Randomly seeded fixed particles. 
Fig. 15 compares the permeability obtained for the randomly (static) fixed particles case and for the dynamic simulations with classic theories for ordered or random arrays of fixed spheres. We can observe that the randomly seeded particles case is very close to Happel's estimate which is suitable for an ordered array of particles. This result is unexpected as we compared a random organization of particles in Poiseuille channel flow to an ordered array in a uniform flow field. The dynamic simulations performed with the Force Coupling Method give the same range of bulk permeability obtained in static simulations only when the compactness is large enough to generate a rather uniform and random packing of particles.

\subsection{Aggregate microstructure and coordination number}

To investigate the structure of the plug, the 3D spherical particles which are fixed in the channel are projected onto the plane $X-Y$. Projections are obtained by integration of a phase indicator $\tau$ (Eq. (34)). If this integration is processed over one spatial coordinate ( $z$-coordinate for example), a gray scale projection $C(\mathbf{x}, \mathbf{y})$ (Eq. (35)) is obtained which is a measure of the particle concentration scanned along the direction of integration (similarly to X-ray tomography).

$\tau=\{0$ in fluid -1 in solid $\}$

$C(\mathbf{x}, \mathbf{y})=\frac{1}{\phi_{0} Z} \int \tau(\mathbf{x}, \mathbf{y}, \mathbf{z}) d \mathbf{z}$

Figs. 16 and 17 show the aggregate structures for an inlet volume fraction equal to $20 \%$. We observe the formation of dendritic structures similarly to Payatakes and Gradon [33] in their study of aerocolloidal particles motion through fiber filters. This evolution of the aggregate is initiated at the walls 1 and 3 . The dendrites progress from the channel walls towards the bulk (Fig. 16) where the fluid velocity is high. These dendritic structures act as a collector and progressively lead to the complete blockage of the channel (Fig. 17). The microstructure is highly heterogeneous.

The coordination number gives information about the number of fixed particles permanently bonded to a reference particle. It is strongly related to the microstructure of an aggregate. It measures the chain or cluster type formed by this particle and its neighbors. A coordination number equal to zero indicates that there is no contact with other particles. This particle is then free to move through the simulation domain or is attached onto a wall. Based on the statistics of the coordination number throughout the suspension, we

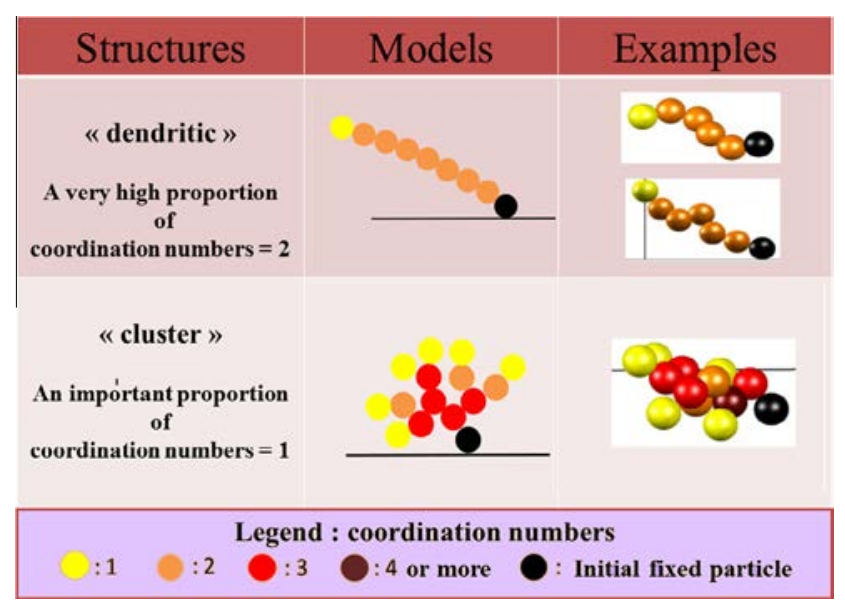

Fig. 18. Coordination number corresponding to distinct aggregate structures.

can identify different aggregate structures shown in Fig. 18. A dendritic structure is characterized by a strong occurrence of coordination numbers equal to 2 . For a cluster structure, a high occurrence of coordination numbers equal to 1 appears for particles on the outer region of the cluster, and 3 within the cluster core.

Without DLVO repulsion force, dendritic structures formed at the beginning of the aggregation process are evolving towards clusters. For simulations at $\phi_{0}=20 \%$, dendritic structures shown in Fig. 16 for 170 attached particles yields coordination numbers with the following distribution: $68 \%$ for $0,6 \%$ for 1 and $26 \%$ for 2 . The transition from dendritic structure to cluster induces the mean coordination number to increase with more occurrence of 3 and 4. For example, the cluster structure presented in Fig. 17 for 320 attached particles induces coordination numbers with the following statistics: $33 \%$ for $0,25 \%$ for $1,20 \%$ for $2,18 \%$ for 3 and $4 \%$ for 4 . Due to rigid and irreversible adhesions of particles when they get into contact the coordination numbers are rarely greater than 4 (the aggregates are open and very loose).

\subsection{Perturbation of the fluid flow}

In this section, we report on the fluid flow associated to plug formation (see a snapshot of the instantaneous velocity field in Fig. 19). Without attached particles, the simulation starts with

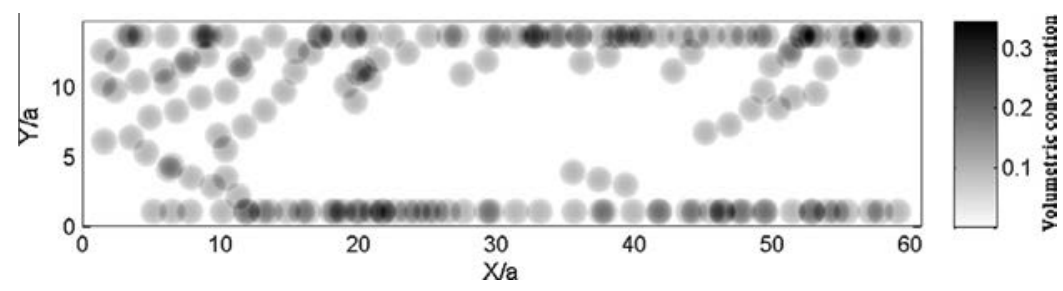

Fig. 16. Side view of the channel with 170 attached particles.

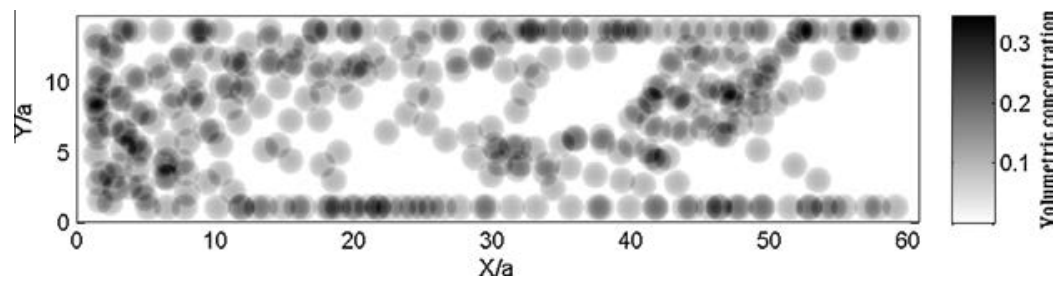

Fig. 17. Side view of the channel with 320 attached particles. 


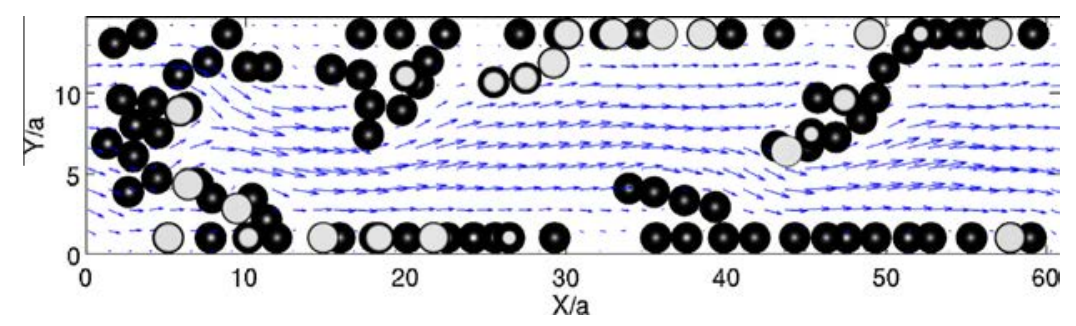

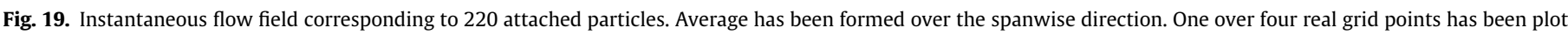
to ease visualization ( 8 grid points fit within a particle diameter).

the classic Poiseuille profile which corresponds to the imposed pressure drop along the channel streamwise direction. While aggregates of particles progressively block the channel, the velocity profile is modified by fixed aggregates. Actually, the formation of aggregates does not occur homogeneously in the channel but depends strongly on the interplay between the fluid velocity (which is responsible for collisions and attachment) and the 3D development of the structure (which perturbs the flow). When a dendrite starts to form on one wall of the channel, it is acting as a collector. Therefore, the structure development is enhanced by new impacting particles and the fluid velocity is reduced which promotes asymmetry of the flow.

Fig. 19 corresponds to fluid flowing through a channel containing 220 attached particles. The flow magnitude is greater around the centerline of the channel where particle aggregates have not formed yet whereas it is equal to zero close to aggregates of fixed particles. The local growing of large particle aggregates causes a significant modification of the fluid flow field.

\section{Channel blockage under repulsive conditions}

Under various physical-chemical conditions clogging may occur although repulsive forces between particles or particles and walls correspond to a stable suspension (aggregation does not occur in a quiescent fluid but particle contact is forced by the flow). Under such conditions, the occurrence and kinetics of plug formation is related to the relative magnitude of hydrodynamic forces (orthokinetic aggregation), repulsive and attractive potentials. In the literature, it has been numerically and experimentally shown that surface interactions like DLVO forces can play a significant role in clogging mechanisms [34]. Aggregation and clogging phenomena for inlet particle concentration $\phi_{0}=10 \%$ will be discussed for different repulsive barrier magnitudes. When DLVO interaction forces are considered, no initial particle adhesion is required. Indeed, the presence of interparticle forces leads to stronger agitation of particles and consequently higher cross-stream self-diffusion. This significantly reduces the time for the first particle-wall adhesions.

\subsection{Magnitude of the repulsion force}

DLVO interparticle forces are the sum of the Van der Waals attraction and an electrical double layer repulsive force due to surface charges. Those forces have been scaled by the typical magnitude of shear induced hydrodynamic force $F_{h}$ (Eq. (36)) which yields contact:

$F_{h}=12 \pi \mu a^{2} \gamma_{0}$

For particle-particle interactions, the magnitude of the attraction force $A_{h} / a$ is constant and equal to $F_{h} / 10$. For particle-wall interaction, $A_{h} / a$ is equal to $F_{h} / 45,000$. The interaction length is kept constant and equal to $0.2 a$ throughout all simulations.
The electrostatic repulsive force has an interaction distance equal to $4 a$ which is fixed by choosing the Debye length. Its relative magnitude is fixed by selecting an appropriate value for $\epsilon_{0} \epsilon_{r} \psi^{2}$ (Eq. (14)). The repulsive force between particles and walls $F_{\mathrm{pw}}$ is set to zero. In the following simulations, only particle-particle interaction force $F_{\mathrm{pp}}$ is varied. Thus, the repulsive force between particles have been selected equal to $F_{h} / 40-F_{h} / 20-F_{h} / 14-F_{h} / 10$ (see Fig. 20). The typical values of $F_{\mathrm{pp}}$ that we selected allows investigating gradually configurations ranging from pure adhesion (previous section) to strong repulsion corresponding to very rare occurrence of particle aggregation. The maximum of repulsion is located at $0.3 a$ while the particle-particle interaction force vanishes for distance equal to $4 a$.

Simulation conditions can represent the behavior of micrometric particle (where Brownian diffusion is negligible) interacting via long-range electrostatic repulsion (having the same order as the particle size). Such colloidal dispersion can be obtained by dispersing micrometric particle in a solvent having low dielectric constant and low salt concentration; Debye length and particle size can then stand in the micrometric range [37].

\subsection{Channel permeability}

Accounting for non-hydrodynamic interaction forces, the simulation results show striking differences for the evolution of the normalized permeability (Fig. 21). Increasing the repulsive force reduces the occurrence of adhesion events and the reduction of permeability is weaker.

Two observations can be made depending on the strength of DLVO repulsive force. The time for first adhesions is shorter when the repulsion is strong. For a normalized volume of injected fluid equal to 0.18 the permeability is equal to 0.60 at $F_{\mathrm{pp}}=F_{h} / 10$ while

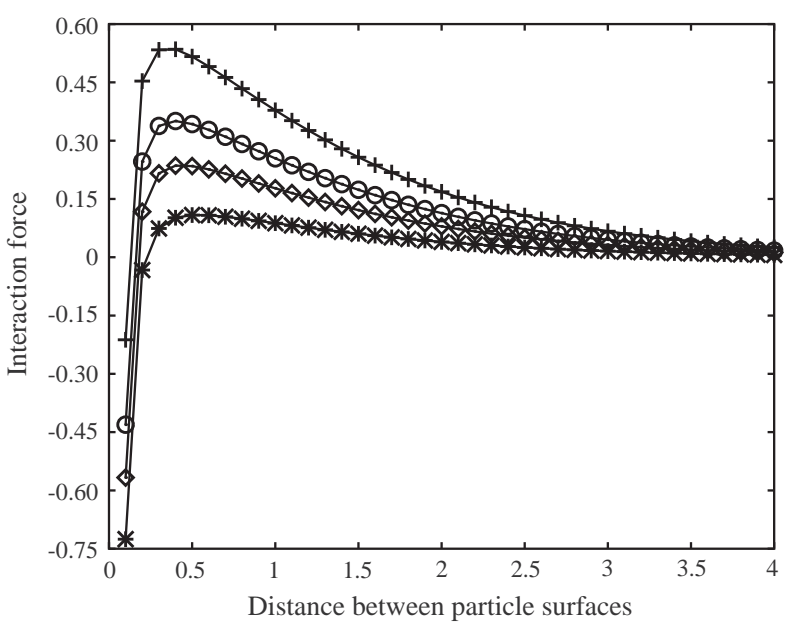

Fig. 20. Profile of DLVO forces (attraction + repulsion) between a pair of particles with different values of $F_{\mathrm{pp}}:-*-F_{h} / 40,-\diamond-F_{h} / 20$, - - $F_{h} / 14,-+-F_{h} / 10$. 


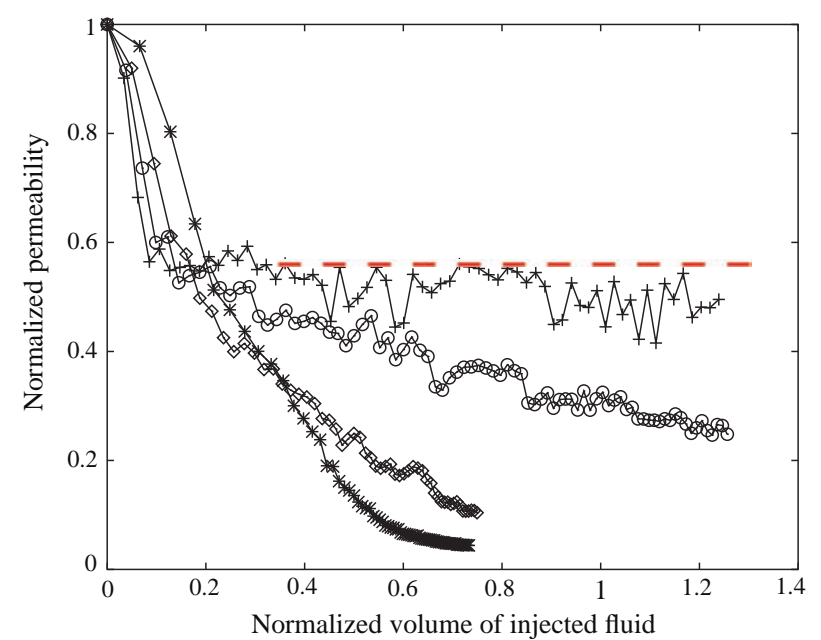

Fig. 21. Normalized permeability vs. normalized volume of injected fluid for different values of $F_{\mathrm{pp}}:-*-F_{h} / 40,-\diamond-F_{h} / 20,-\circ-f_{h} / 14,-+-F_{h} / 10$. The dashed line corresponds to the permeability with a monolayer of attached particles.

it is equal to 0.82 at $F_{\mathrm{pp}}=F_{h} / 40$. Da Cunha and Hinch [36] clearly demonstrated that shear induced self-diffusion is function of the strength of repulsive forces which yield a net displacement at each pairwise particle interaction. Particles are moving across streamlines enhancing cross-stream diffusion (adhesion to wall occurs sooner). The second observation is concerned with the temporal evolution of the permeability. When repulsion is weak $F_{\mathrm{pp}}=F_{h} / 40$, the permeability evolution is similar to purely adhesive case $F_{\mathrm{pp}}=0$.

The situation is dramatically different for strong repulsion $\left(F_{\mathrm{pp}}=F_{h} / 10\right)$. The normalized permeability decreases due to particles adhesion onto the walls and reaches a plateau slightly below 0.58 (dashed line in Fig. 21 corresponding to a monolayer of attached particles). Under constant pressure drop, the decrease of permeability corresponds to flowrate reduction and consequently wall shear reduction, as well. Below a critical value, shear can no longer provokes adhesion of particles on the wall particle monolayer. This phenomenon leads to a nearly constant permeability. The permeability temporal evolution for $F_{\mathrm{pp}}=F_{h} / 10$ is fluctuating (Fig. 21 - curve - +-). An explanation based on dynamic visualization of particle snapshots consists in a scenario where particles form a network due to repulsive forces (gel like transition). Under hydrodynamic forcing this network resists (slight decrease of permeability) and suddenly, the rearrangement of particles leads to a rapid (but moderate) increase of permeability. This phenomenon recovers as particles progress towards the exit of the channel.

In the absence of DLVO forces, the force monopole acting on flowing particles is set to zero while it is counterbalancing adhesion force for fixed particles. The presence of non-hydrodynamic interaction forces (particularly repulsion force) modifies this simple picture while attached and freely moving particles experienced interactions with neighbors. In Fig. 22, the probability distribution function of the normalized force monopole at $F_{\mathrm{pp}}=F_{h} / 10$ shows that both fixed and freely moving particles experience fluid or interparticle forces. The simulation time chosen for Fig. 22 corresponds to one instant of fluctuations observed in Fig. 21. For attached particles the force monopole is positive which corresponds to the adhesive contribution of the contact forces. The force acting on the particles is directed downstream. For flowing particles, we note that positive and negative values of interaction forces are possible. Actually, the intensity and the direction of the force monopole acting on each particle may change frequently due to the relative motion of particles through the channel.

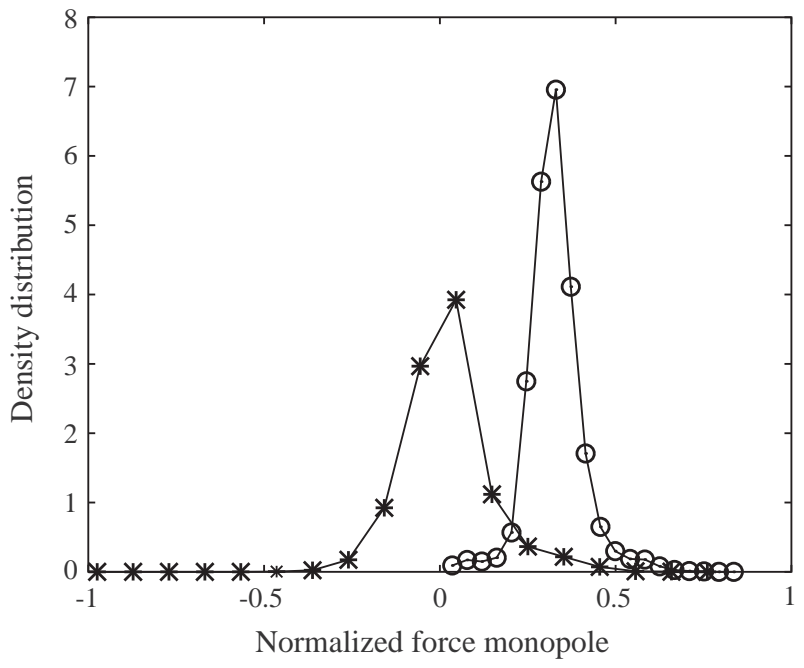

Fig. 22. PDF of force monopole for $\phi_{0}=10 \%$ and $F_{\mathrm{pp}}=F_{h} / 10$ at $t_{s}=38.8$, -*flowing particles, -०- attached particles.

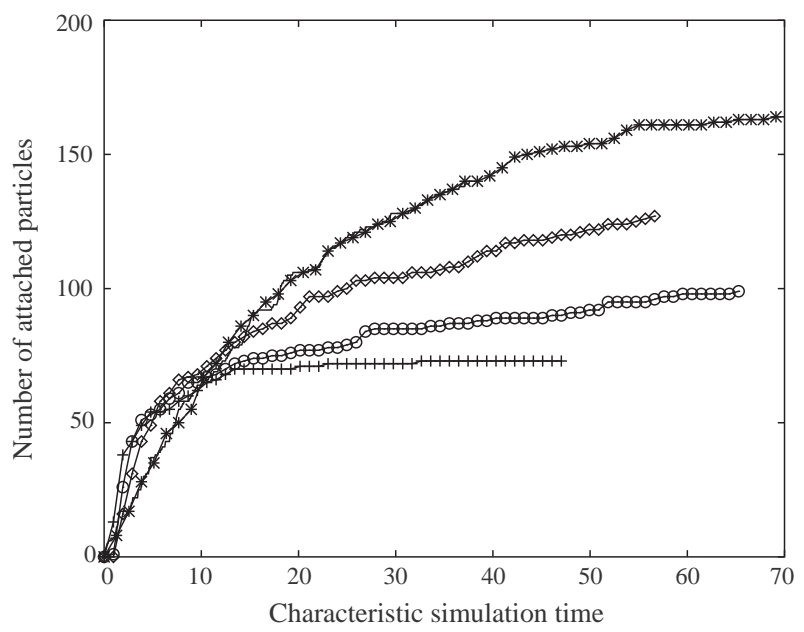

Fig. 23. Temporal evolution of the number of captured particles for different values of $F_{\mathrm{pp}}:-0,-*-F_{h} / 40,-\diamond-F_{h} / 20,-\circ-F_{h} / 14,-+-F_{h} / 10$. Cases $F_{\mathrm{pp}}=0$ and $F_{\mathrm{pp}}=F_{h} / 40$ are closely superposed.

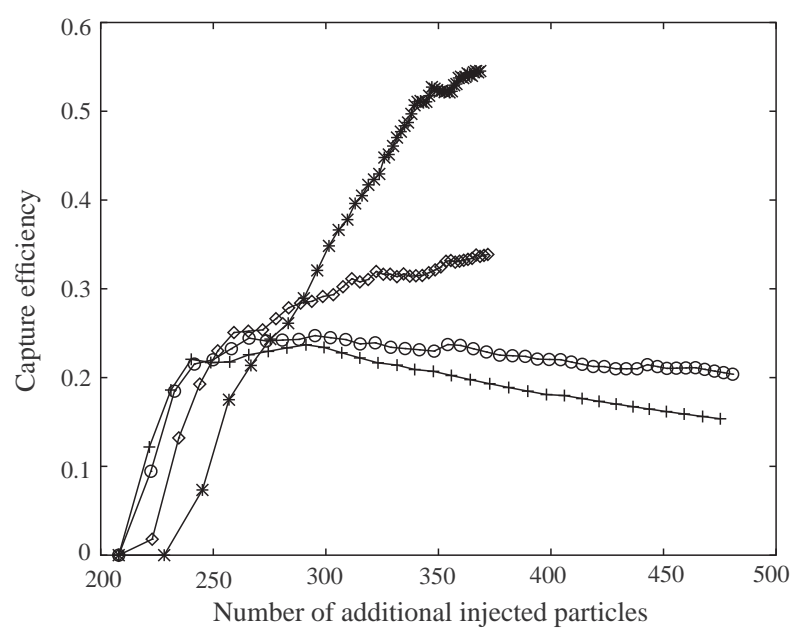

Fig. 24. Evolution of capture efficiency vs. number of injected particles for different values of $F_{\mathrm{pp}}:-*-F_{h} / 40,-\diamond-F_{h} / 20,-\circ-F_{h} / 14,-+-F_{h} / 10$. 


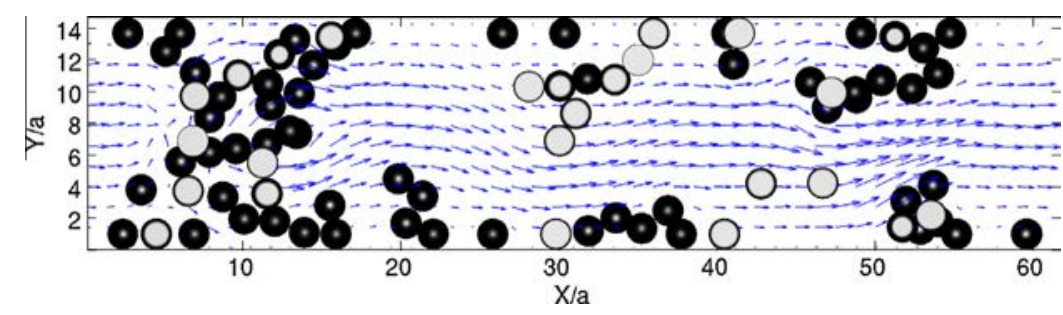

Fig. 25. Instantaneous velocity flow field associated with 200 attached particles for $F_{\mathrm{pp}}=F_{h} / 40$.

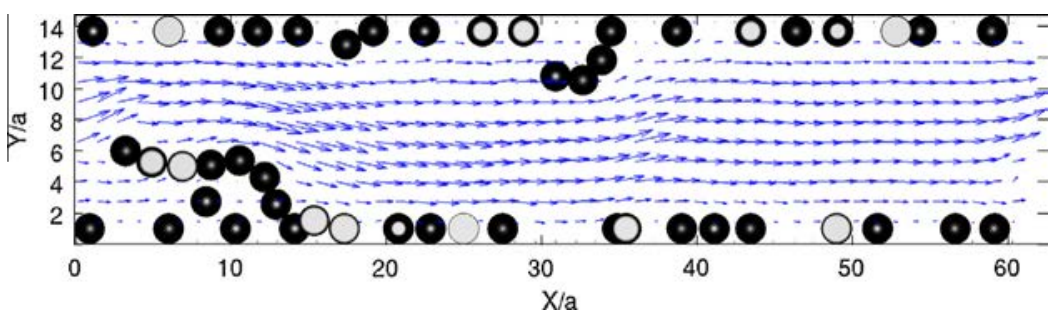

Fig. 26. Instantaneous velocity flow field associated with 100 attached particles for $F_{\mathrm{pp}}=F_{h} / 14$.

\subsection{Dynamics of particle adhesion and capture efficiency}

Channel permeability is strongly related to the number of attached particles. The temporal evolution of the number of fixed particles is presented in Fig. 23. At a characteristic simulation time $t_{s}$ equal to 30 , the number of captured particles (which is directly given by incremental counting of new attached particles) is equal to $148,104,85$ and 72 for $F_{\text {pp }}$ equal to $F_{h} / 40, F_{h} / 20, F_{h} / 14$ and $F_{h} / 10$ respectively. Although first adhesions occur sooner with high repulsive forces $F_{\mathrm{pp}}=F_{h} / 10$, the number of attached particles reaches a plateau. Comparing the simulation results obtained for weak repulsion to the case of purely adhesive particles, it is clear that the dynamics of capture is similar. For $F_{\mathrm{pp}}=F_{h} / 10$, the number of attached particles stabilizes at 72 while time goes on and new particles are continuously injected in the channel. Those 72 particles correspond to adhesion to walls resulting from successive repulsions and drift through the bulk flow.

The capture efficiency is defined as the ratio of the number $N_{\text {cap }}$ of fixed particles to the number $N_{\text {inj }}$ of injected particles (Eq. (37)) through the inlet section of the microchannel.

$N_{\text {inj }}=\frac{\phi_{0} S}{V_{p}} \int_{0}^{T} u_{\text {mean }} d t$

$V_{p}$ represents the particle volume, $\phi_{0}$ the particle inlet concentration. $S$ is the area of the channel cross section and $S u_{\text {mean }}$ is the average flowrate.

The evolution of the capture efficiency is presented in Fig. 24 where two distinct behaviors are observed. For $F_{\mathrm{pp}}=F_{h} / 40$, capture efficiency increases while particles are injected in the channel. For 275 injected particles, the capture efficiency is equal to 0.28 for $F_{\mathrm{pp}}=F_{h} / 40$ while it has already reached 0.47 in the absence of repulsive forces. Adding repulsive forces can change dramatically the long term evolution of the capture efficiency. Progressively increasing repulsive force, leads to a non-intuitive response of the system. At $F_{\mathrm{pp}}=F_{h} / 10$ for example, the capture efficiency has two distinct trends. The first one corresponds to an increase of capture efficiency associated with wall adhesions. The second trend is a monotonous decrease: capture efficiency is equal to 0.25 for 250 injected particles and then decreases down to 0.16 for 450 injected particles. Injection of new particles through the channel inlet does not lead to any additional adhesions of particles. The number of attached particles is stabilized. New particles are not able to overcome the repulsive barrier preventing adhesion and cluster formation. Consequently, this leads to the decrease of capture efficiency.

\subsection{Aggregate structures and flow perturbation}

The 3D structure of aggregates formed by fixed particles is drastically different in the presence of repulsive force. For low repulsion forces $\left(F_{\mathrm{pp}}=F_{h} / 40\right)$, clusters of particles observed in Fig. 25 are similar to purely adhesive case. The statistics of coordination number are dominated by occurrence of one and three neighbors. With strong interaction repulsive forces $\left(F_{\mathrm{pp}}=F_{h} / 10\right)$, only particle monolayers attached onto the wall are observed. New particles are not able to overcome the repulsive barrier preventing adhesion and cluster formation. Therefore, the coordination number characterizing this situation is equal to zero. For moderate repulsion strength $\left(F_{\mathrm{pp}}=F_{h} / 14\right)$, particles aggregate form chainlike structures (Fig. 26). Early adhesion events yield a particle monolayer similar to what was obtained for $F_{\mathrm{pp}}=F_{h} / 10$. Later on, chainlike structures appear in the channel resulting from an interplay between the hydrodynamic forcing and repulsive force barriers preventing the formation of clusters. The chainlike structure is characterized by a distribution of coordination number dominated by two neighbors.

\section{Conclusion}

In this paper, we investigated the behavior of flowing suspensions in a microchannel. The Force Coupling Method is an efficient and flexible way to account for hydrodynamic multibody interactions together with non-hydrodynamic interparticle forces (particularly DLVO forces including adhesion effects and repulsion barriers to model particle-particle and particle-wall interactions). The set of simulations performed at constant pressure drop in absence of DLVO forces show that dendritic structures are initially formed from the wall and lead to the progressive blockage of the channel. The variation in time of the permeability does not follow the classical theories of filtration in packed beds because the development of the plug is not homogeneous across the channel width. The volume fraction of particles in the dispersion has a minor effect on the variation of the normalized permeability and on the aggregate microstructure. The reduction of permeability is mainly controlled by the number of attached particles as time progresses. 
For simulations including the effect of DLVO interaction forces, we showed that the evolution of the normalized permeability depends strongly on the strength of the repulsion barrier. An increase of particle-particle interaction force induces a reduction of the number of attached particles and consequently higher permeability. The fluctuations observed in the evolution of the normalized permeability at a strong repulsion are due to rearrangement of flowing particles interconnected by DLVO forces. Clearly, this contributes to the permeability reduction at high concentration while non-adhesive cluster of particles has to move towards the exit while interacting with fixed particles on walls. We observed the transition from cluster structures characteristic of purely adhesive suspension to the formation of particle monolayer for strong repulsion barrier. At intermediate values of repulsive inter-particle forces, chainlike structures appear.

This study showed the important role played by hydrodynamic and non-hydrodynamic (DLVO) interaction forces in the process of particle aggregation and straight microchannel clogging. The analysis of particle deposition through non-homogeneous porous surfaces (for instance a pore entrance) would lead to significant enhancement of basic features of filtration membrane fouling.

\section{References}

[1] Climent E, Maxey MR. The force coupling method: a flexible approach for the simulation of particulate flows. In: Feuillebois F, Sellier A, editors. Methods for creeping flows. Ressign Press; 2010.

[2] Hermia J. Constant pressure blocking filtration laws-application to power-law non-newtonian fluids. Chem Eng Res Des 1982;60:183-7.

[3] Happel J. Viscous flow in multiparticle systems, slow motion of fluids relative to beds of spherical particles. AiChE J 1958;4:197.

[4] Sharp KV, Adrian RJ. On flow-blocking particle structures in microtubes. Microfluid Nanofluid 2005;1:376-80.

[5] Wyss MH, Blair LD, Morris FJ, Stone AH, Weitz AD. Mechanism for clogging of microchannels. Phys Rev 2006;74:061402.

[6] Ramachandran V, Fogler HS. Plugging by hydrodynamic bridging during flow of stable colloidal particles within cylindrical pores. J Fluid Mech 1999;385:129-56.

[7] Marshall JS. Particle aggregation and capture by walls in a particulate aerosol channel flow. Aerosol Sci 2007;38:333-51.

[8] Tanaka H, Araki T. Simulation method of colloidal suspensions with hydrodynamic interactions. Phys Rev Lett 2000;45:6.

[9] Lomholt S, Maxey MR. Force coupling method for particulate two-phase flow: Stokes flow. J Comput Phys 2003;184:381-405.

[10] Agbangla GC, Climent E, Bacchin P. Experimental investigation of pore clogging by microparticles: evidence for a critical flux density of particle yielding arches and deposits. Sep Purif Technol 2012;101:42-8.

[11] Abbas M, Climent E, Simonin O, Maxey M. Dynamics of bidisperse suspensions under Stokes flows: linear shear flow and sedimentation. Phys Fluids 2006:18:121504.

[12] Pozrikidis C. Boundary Integral and Singularity Methods for Linearized Viscous Flow. Cambridge University Press; 1992. ISBN 0521406935.

[13] Happel J, Brenner H. Low Reynolds Number Hydrodynamics. The Hague: Marinus Nijhoff Publishers; 1967.

[14] Brady JF, Bossis G. Stokesian dynamics. Ann Rev Fluid Mech 1998;20:111-57.
[15] Sierou A, Brady FF. Accelerated Stokesian dynamics simulations. J Fluid Mech 2001:48:115-46.

[16] Climent E, Maxey MR. Numerical simulations of random suspensions at finite Reynolds number. Int J Multiphase Flows 2003;29:579-601.

[17] Dance SL, Maxey MR. Incorporation of lubrication effects into the force coupling method for particulate two-phase flow. J Comput Phys 2003;189:212-38.

[18] Lomholt S, Stenum B, Maxey MR. Experimental verification of the force coupling method for particle flows. Int J Multiphase 2002;28:225-46.

[19] Maxey MR, Patel BK. Localized force representations for particles sedimenting in Stokes flow. Int J Multiphase Flow 2001;27:1603-26.

[20] Peskin C. The immersed boundary method. Acta Numer 2002:11:1-39.

[21] You CF, Li HG, Qi YH, Xu XC. Motion of micro-particles in channel flow. J Atmos Environ 2004;38:1559-65.

[22] Ganatos P, Weinbaum S, Pfeffer R. A strong interaction theory for the creeping motion of a sphere between plane parallel boundaries. Part 2. Parallel motion. J Fluid Mech 1980;99:755-83.

[23] Sangani AS, Acrivos A. Slow flow through a periodic array of spheres. J Multiphase Flow 1982:8:343-60.

[24] Bhattacharya S, Blawzdziewicz J, Wajnryb E. Hydrodynamic interactions of spherical particles in Poiseuille flow between two parallel walls. Phys Fluids 2006;18:053301.

[25] Feke DL, Schowalter WR. The effect of Brownian diffusion on shear-induced coagulation of colloidal dispersions. J Fluid Mech 1983;133:17-35.

[26] Drazer G, Koplik J, Khusid B, Acrivos A. Microstructure and velocity fluctuations in sheared suspensions. J Fluid Mech 2004:511:237-63.

[27] Zick AA, Homsy GM. Stokes flow through periodic arrays of spheres. J Fluid Mech 1982;115:13.

[28] Richardson JF, Zaki WN. Sedimentation and fluidization, Part I. Trans Inst Chem Eng 1954;32:35.

[29] Batchelor GK. Sedimentation in a dilute dispersion of spheres. J Fluid Mech 1972;52:45-268.

[30] Kim S, Russel WS. Modelling of porous media by renormalization of the Stokes equations. J Fluid Mech 1985;154:269-86.

[31] Brian GP, Velev OD. Controlled, rapid deposition of structured coatings from micro and nano particle suspensions. Langmuir 2004;20:2099-107.

[32] Mustin B, Stoeber B. Deposition of particles from polydisperse suspensions in microfluidic systems. Microfluid Nanofluid 2003;9:905-13.

[33] Payatakes AC, Gradon L. Dendritic deposition of aerosol by convective Brownian diffusion for small, intermediate and high particle Knudsen numbers. AIChE J 1980;26:443-54.

[34] Unni HN, Yang C. Brownian dynamics simulation and experimental study of colloidal particle deposition in microchannel flow. Adv Colloid Interface Sci 2005;291:28-36.

[35] Yeo K, Maxey MR. Simulation of concentrated suspensions using the forcecoupling method. J Comput Phys 2010;229:2401-21.

[36] Da Cunha FR, Hinch EJ. Shear-induced dispersion in a dilute suspension of rough spheres. J Fluid Mech 1996;309:211-23.

[37] El Masri D, Vissers T, Badaire S, Stiefelhagen JP, Rao Vutukuri H, Helfferich P, et al. A qualitative confocal microscopy study on a range of colloidal processes by simulating microgravity conditions through slow rotations. Soft Matter 2012;8:6979.

[38] Henry C, Minier JP, Lefévre G. Towards a description of particulate fouling: from single particle deposition to clogging. Adv Colloid Interface Sci 2012;185-186:34-76.

[39] Mohaupt M, Minier JP, Taniére A. A new approach for the detection of particle interactions for large-inertia and colloidal particles in a turbulent flow. Int J Multiphase Flow 2011;37:746-55.

[40] Dong S, Liu D, Maxey MR, Karniadakis GE. Spectral distributed Lagrange multiplier method: algorithm and benchmark tests. J Comput Phys 2004; 195:695-717. 